\title{
PITT'S INEQUALITY AND THE FRACTIONAL LAPLACIAN: SHARP ERROR ESTIMATES
}

\author{
for Eli Stein
}

\author{
WILLIAM BECKNER
}

\begin{abstract}
Sharp error estimates in terms of the fractional Laplacian and a weighted Besov norm are obtained for Pitt's inequality by using the spectral representation with weights for the fractional Laplacian due to Frank, Lieb and Seiringer and the sharp Stein-Weiss inequality. Dilation invariance, group symmetry on a non-unimodular group and a nonlinear Stein-Weiss lemma are used to provide short proofs of the Frank-Seiringer "Hardy inequalities" where fractional smoothness is measured by a Besov norm.
\end{abstract}

Considerable interest exists in understanding the framework of weighted inequalities for differential operators and the Fourier transform, and the application of quantitative information drawn from these inequalities to varied problems in analysis and mathematical physics, including nonlinear partial differential equations, spectral theory, fluid mechanics, stability of matter, stellar dynamics and uncertainty. Such inequalities provide both refined size estimates for differential operators and singular integrals and quantitative insight on symmetry invariance and geometric structure. The purpose of this note is to improve the sharp Pitt's inequality at the spectral level by using the optimal Stein-Weiss inequality and a new representation formula derived by Frank, Lieb and Seiringer [19] which expresses fractional Sobolev embedding in terms of a Besov norm characteristic of the problem's dilation invariance and extends with weights an earlier classical formula of Aronszajn and Smith using the $L^{2}$ modulus of continuity (see [1], page 402). Moreover reflecting the natural duality, this formula can be combined with the Hardy-Littlewood-Sobolev inequality to provide new techniques to determine sharp embedding constants, including a sharp form of the Besov norm Sobolev embedding studied by Bourgain, Brezis and Mironescu [16. A secondary bootstrap argument produces an improved Stein-Weiss inequality accompanied by an intriguing monotonicity property at the spectral level. A direct proof of the weighted representation formula for the fractional Laplacian starting from the classical formula of Aronszajn and Smith is given in the appendix below. This classical formula provides an alternative way to view Pitt's inequality and Hardy inequalities in terms of fractional smoothness and Besov norms with natural extensions to $L^{p}$ classes. In this context, a new Stein-Weiss lemma, unusual in its simplicity, is applied to obtain the Frank-Seiringer "Hardy inequalities" for both $\mathbb{R}^{n}$ and the upper half-space $\mathbb{R}_{+}^{n}[20,21$, and corresponding extensions for the Heisenberg group and product spaces with mixed homogeneity.

\section{Pitt's inequality.}

This paper's principle result is the following theorem which gives specific improvement by a Besov norm for Pitt's inequality and explicitly demonstrates that the sharp constant is not attained which otherwise was observed from the equivalent Young's inequality (see [6]). 
Theorem 1. For $f \in \mathcal{S}\left(\mathbb{R}^{n}\right)$ and $0<\beta<2, \beta \leq \alpha<n$

$$
\begin{aligned}
C_{\alpha} \int_{\mathbb{R}^{n}}|\xi|^{\alpha}|\hat{f}|^{2} d \xi \geq \int_{\mathbb{R}^{n}}|x|^{-\alpha}|f|^{2} d x & \\
& \quad+\frac{C_{\alpha}}{D_{\beta}} \int_{\mathbb{R}^{n} \times \mathbb{R}^{n}} \frac{|g(x)-g(y)|^{2}}{|x-y|^{n+\beta}}|x|^{-(n-\beta) / 2}|y|^{-(n-\beta) / 2} d x d y
\end{aligned}
$$

where

$$
g(x)=|x|^{(n-\beta) / 2}\left(-\Delta / 4 \pi^{2}\right)^{(\alpha-\beta) / 4} f(x)
$$

and

$$
C_{\alpha}=\pi^{\alpha}\left[\frac{\Gamma\left(\frac{n-\alpha}{4}\right)}{\Gamma\left(\frac{n+\alpha}{4}\right)}\right]^{2}, \quad D_{\beta}=\frac{4}{\beta} \pi^{\frac{n}{2}+\beta} \frac{\Gamma\left(1-\frac{\beta}{2}\right)}{\Gamma\left(\frac{n+\beta}{2}\right)} .
$$

Before outlining the proof for this result, the context for the problem will be described. Note that equation (11) becomes an equality if $\alpha=\beta$, the Fourier transform is defined by

$$
(\mathcal{F} f)(\xi)=\hat{f}(\xi)=\int_{\mathbb{R}^{n}} e^{2 \pi i \xi x} f(x) d x
$$

primes denote dual exponents, $1 / p+1 / p^{\prime}=1$, and $\Delta$ is the Laplacian on $\mathbb{R}^{n}$.

The framework for this development lies at the interface of two classical results combined with the recent representation formula for the fractional Laplacian obtained by Frank, Lieb and Seiringer: namely, Pitt's inequality which may be viewed as a weighted extension of the Hausdorff-Young inequality and the Stein-Weiss inequality which extends the Hardy-LittlewoodSobolev inequality for fractional integrals and in effect Young's inequality for convolution thus reinforcing the natural operator duality. This paper is the fourth in a series [6, 12, 13] which was originally motivated by an effort to better understand how the classical uncertainty principle could be extended to incorporate the logarithmic Sobolev inequality. In a larger sense, the purpose is to determine the implications of dilation invariance on the Euclidean manifold.

Pitt's inequality. For $f \in \mathcal{S}\left(\mathbb{R}^{n}\right), 1<p \leq q, \infty, 0 \leq \alpha<n / q, 0 \leq \beta<n / p^{\prime}$ and $n \geq 2$

$$
\left[\left.\left.\int_{\mathbb{R}^{n}}|| x\right|^{-\alpha} \hat{f}\right|^{q} d x\right]^{1 / q} \leq A\left[\left.\left.\int_{\mathbb{R}^{n}}|| x\right|^{\beta} f\right|^{p} d x\right]^{1 / p}
$$

with the index constraint

$$
\frac{n}{p}+\frac{n}{q}+\beta-\alpha=n
$$

Pitt's inequality (at the spectral level). For $f \in \mathcal{S}\left(\mathbb{R}^{n}\right)$ and $0 \leq \alpha<n$

$$
\begin{gathered}
\int_{\mathbb{R}^{n}}|x|^{-\alpha}|f(x)|^{2} d x \leq C_{\alpha} \int_{\mathbb{R}^{n}}|\xi|^{\alpha}|\hat{f}(\xi)|^{2} d \xi \\
C_{\alpha}=\pi^{\alpha}\left[\Gamma\left(\frac{n-\alpha}{4}\right) / \Gamma\left(\frac{n+\alpha}{4}\right)\right]^{2}
\end{gathered}
$$

Stein-Weiss inequality. For $f \in L^{p}\left(\mathbb{R}^{n}\right)$ with $1<p<\infty, 0<\lambda<n, \alpha<n / p, \beta<n / p^{\prime}$ and $n=\lambda+\alpha+\beta$

$$
\begin{gathered}
\left\||x|^{-\alpha}\left(|x|^{-\lambda} *\left(|x|^{-\beta} f\right)\right)\right\|_{L^{p}\left(\mathbb{R}^{n}\right)} \leq E_{\alpha, \beta}\|f\|_{L^{p}\left(\mathbb{R}^{n}\right)} \\
E_{\alpha, \beta}=\pi^{n / 2}\left[\frac{\Gamma\left(\frac{\alpha+\beta}{2}\right) \Gamma\left(\frac{n}{2 p}-\frac{\alpha}{2}\right) \Gamma\left(\frac{n}{2 p^{\prime}}-\frac{\beta}{2}\right)}{\Gamma\left(\frac{n-\alpha-\beta}{2}\right) \Gamma\left(\frac{n}{2 p^{\prime}}+\frac{\alpha}{2}\right) \Gamma\left(\frac{n}{2 p}+\frac{\beta}{2}\right)}\right]
\end{gathered}
$$


Note that it is not required that both $\alpha$ and $\beta$ are non-negative, but rather $0<\alpha+\beta<n$.

Spectral representation for the fractional Laplacian (Frank-Lieb-Seiringer). For $f \in \mathcal{S}\left(\mathbb{R}^{n}\right)$ and $0 \leq \alpha<\min \{2, n\}$

$$
\begin{gathered}
C_{\alpha} \int_{\mathbb{R}^{n}}|\xi|^{\alpha}|\hat{f}(\xi)|^{2} d \xi=\int_{\mathbb{R}^{n}}|x|^{-\alpha}|f(x)|^{2} d x \\
+D_{\alpha} \int_{\mathbb{R}^{n} \times \mathbb{R}^{n}} \frac{|g(x)-g(y)|^{2}}{|x-y|^{n+\alpha}}|x|^{-(n-\alpha) / 2}|y|^{-(n-\alpha) / 2} d x d y
\end{gathered}
$$

where

$$
\begin{gathered}
g(x)=|x|^{(n-\alpha) / 2} f(x) \\
C_{\alpha}=\pi^{\alpha}\left[\Gamma\left(\frac{n-\alpha}{4}\right) / \Gamma\left(\frac{n+\alpha}{4}\right)\right]^{2}, \\
D_{\alpha}=\pi^{-n / 2-\alpha} \frac{\alpha}{4} \frac{\Gamma\left(\frac{n+\alpha}{2}\right)}{\Gamma\left(\frac{2-\alpha}{2}\right)} C_{\alpha} .
\end{gathered}
$$

Observe that in the limit $\alpha \rightarrow 2$, one obtains the classical relation which includes Hardy's inequality

$$
\int_{\mathbb{R}^{n}}|\nabla f|^{2} d x=\frac{(n-2)^{2}}{4} \int_{\mathbb{R}^{n}}|x|^{-2}|f(x)|^{2} d x+\int_{\mathbb{R}^{n}}\left|\nabla\left(|x|^{(n-2) / 2} f\right)\right|^{2}|x|^{-n+2} d x
$$

Proof of Theorem [1. Use the ground state representation (5) to write for $\alpha>\beta$

$$
\begin{aligned}
& C_{\beta} \int_{\mathbb{R}^{n}}|\xi|^{\beta}\left[|\xi|^{\alpha-\beta}|\hat{f}|^{2}\right] d \xi=\int_{\mathbb{R}^{n}}|x|^{-\beta}\left|\mathcal{F}^{-1}\left(|\xi|^{(\alpha-\beta) / 2} \hat{f}\right)\right|^{2} d x \\
& \quad+D_{\beta} \int_{\mathbb{R}^{n} \times \mathbb{R}^{n}} \frac{|g(x)-g(y)|^{2}}{|x-y|^{n+\beta}}|x|^{-(n-\beta) / 2}|y|^{-(n-\beta) / 2} d x d y
\end{aligned}
$$

where

$$
g(x)=|x|^{(n-\beta) / 2}\left(-\Delta / 4 \pi^{2}\right)^{(\alpha-\beta) / 4} f(x) .
$$

To complete the proof, it remains to show that

$$
\frac{C_{\beta}}{C_{\alpha}} \int|x|^{-\alpha}|f|^{2} d x \leq \int_{\mathbb{R}^{n}}|x|^{-\beta}\left|\mathcal{F}^{-1}\left(|\xi|^{(\alpha-\beta) / 2} \hat{f}\right)\right|^{2} d x .
$$

But this is equivalent to showing the Stein-Weiss inequality

$$
\left.\left.\int_{\mathbb{R}^{n}}|x|^{-\alpha}|| x\right|^{-n+(\alpha-\beta) / 2} *\left(|x|^{\beta / 2} f\right)\right|^{2} d x \leq F_{\alpha, \beta} \int_{\mathbb{R}^{n}}|f|^{2} d x
$$

with

$$
\begin{gathered}
F_{\alpha, \beta}=\frac{C_{\alpha}}{C_{\beta}} \pi^{n-\alpha+\beta}\left[\frac{\Gamma\left(\frac{\alpha-\beta}{4}\right)}{\Gamma\left(\frac{2 n-\alpha+\beta}{4}\right)}\right]^{2} \\
=\pi^{n}\left[\frac{\Gamma\left(\frac{\alpha-\beta}{4}\right) \Gamma\left(\frac{n-\alpha}{4}\right) \Gamma\left(\frac{n+\beta}{4}\right)}{\Gamma\left(\frac{2 n-\alpha+\beta}{4}\right) \Gamma\left(\frac{n+\alpha}{4}\right) \Gamma\left(\frac{n-\beta}{4}\right)}\right]^{2} .
\end{gathered}
$$


This constant exactly matches the bound given by the Stein-Weiss inequality (4) though note the change in notation and that here it is a positive power of $|x|$ multiplying the function $f$. Then

$$
D_{\alpha, \beta}=C_{\alpha} D_{\beta} / C_{\beta}=\pi^{-n / 2+\alpha-\beta} \frac{\beta}{4} \frac{\Gamma\left(\frac{n+\beta}{2}\right)}{\Gamma\left(\frac{2-\beta}{2}\right)}\left[\frac{\Gamma\left(\frac{n-\alpha}{4}\right)}{\Gamma\left(\frac{n+\alpha}{4}\right)}\right]^{2} .
$$

This completes the proof of Theorem 1 .

As evident from the proof outlined above and the previous determination of sharp constants for Pitt's inequality, there is a self-evident duality between Pitt's inequality and the Stein-Weiss inequality. Using

$$
\mathcal{F}\left[|x|^{-\alpha / 2}\right]=\sqrt{d_{\alpha}}|x|^{-n+\frac{\alpha}{2}}, \quad d_{\alpha}=\pi^{-(n-\alpha)}\left[\frac{\Gamma\left(\frac{2 n-\alpha}{4}\right)}{\Gamma\left(\frac{\alpha}{4}\right)}\right]^{2}
$$

then the following error estimate for the Stein-Weiss inequality is obtained from Theorem 1

Theorem 2. For $f \in L^{2}\left(\mathbb{R}^{n}\right)$ and $0<\beta<2, \beta \leq \alpha<n$

$$
\begin{aligned}
\int_{\mathbb{R}^{N}}|f|^{2} d x \geq \frac{d_{\alpha}}{C_{\alpha}} & \left.\left.\int_{\mathbb{R}^{n}}|x|^{-\alpha}|| x\right|^{-n+\frac{\alpha}{2}} * f\right|^{2} d x \\
& +\frac{d_{\beta}}{D_{\beta}} \int_{\mathbb{R}^{n} \times \mathbb{R}^{n}} \frac{|h(x)-h(y)|^{2}}{|x-y|^{n+\beta}}|x|^{-(n-\beta) / 2}|y|^{-(n-\beta) / 2} d x d y .
\end{aligned}
$$

where

$$
h(x)=\left(|x|^{-n+\frac{\beta}{2}} * f\right)(x) .
$$

The expression explicitly demonstrates that the sharp constant for the Stein-Weiss inequality is not attained. Using the Frank-Lieb-Seiringer representation formula, one obtains the following monotonicity result:

Corollary. For $0<\alpha<2$

$$
\left.\left.\frac{d_{\alpha}}{C_{\alpha}} \int_{\mathbb{R}^{n}}|x|^{-\alpha}|| x\right|^{-n+\frac{\alpha}{2}} * f\right|^{2} d x
$$

is monotone decreasing as a function of $\alpha$ !

A natural objective in transforming weighted inequalities for the Fourier transform to convolution estimates is to enable extensions to $L^{p}\left(\mathbb{R}^{n}\right)$ (see [13] where this aspect was the central direction). The classical Aronszajn-Smith representation formula (see Appendix in this paper) allows Theorem 1 to be recast in a form that is amenable to analysis on $L^{p}\left(\mathbb{R}^{n}\right)$ (see section 4 below).

Theorem 3. For $f \in \mathcal{S}\left(\mathbb{R}^{n}\right)$ and $0<\beta<2, \beta \leq \alpha<n$

$$
\begin{aligned}
& \frac{C_{\alpha}}{D_{\beta}} \int_{\mathbb{R}^{n} \times \mathbb{R}^{n}} \frac{|h(x)-h(y)|^{2}}{|x-y|^{n+\beta}} d x d y \geq \int_{\mathbb{R}^{n}}|x|^{-\alpha}|f|^{2} d x \\
& \quad+\frac{C_{\alpha}}{D_{\beta}} \int_{\mathbb{R}^{n} \times \mathbb{R}^{n}} \frac{|g(x)-g(y)|^{2}}{|x-y|^{n+\beta}}|x|^{-(n-\beta) / 2}|y|^{-(n-\beta) / 2} d x d y
\end{aligned}
$$

with $h(x)=|x|^{-(n-\beta) / 2} g(x)=\left(-\Delta / 4 \pi^{2}\right)^{(\alpha-\beta) / 4} f(x)$. 


\section{Logarithmic uncertainty.}

The central motivation for computing optimal constants for Pitt's inequality in [6] was to directly strengthen the classical uncertainty principle. Beginning with analysis of the log Sobolev inequality, one understands that a parameter-dependent variational inequality which becomes an equality at a given parameter value can be differentiated at that value to give a new analytic inequality. Using Pitt's inequality, one obtains (see [6], [13]):

Logarithmic uncertainty. For $f \in \mathcal{S}\left(\mathbb{R}^{n}\right)$ and $1<p<\infty$

$$
\begin{gathered}
\int_{\mathbb{R}^{n}} \ln |x||f|^{2} d x+\int_{\mathbb{R}^{n}} \ln |\xi||\hat{f}|^{2} d \xi \geq D \int_{\mathbb{R}^{n}}|f|^{2} d x \\
D=\psi(n / 4)-\ln \pi, \quad \psi=(\ln \Gamma)^{\prime} \\
\int_{\mathbb{R}^{n}} \ln |x||f|^{p} d x+\int_{\mathbb{R}^{n}}\left[\left(\ln \sqrt{\left(-\Delta / 4 \pi^{2}\right)}\right) f\right] f|f|^{p-2} d x \geq E \int_{\mathbb{R}^{n}}|f|^{p} d x \\
E=\frac{1}{2}\left[\psi(n / 2 p)+\psi\left(n / 2 p^{\prime}\right)\right]-\ln \pi
\end{gathered}
$$

Since the Frank-Lieb-Seiringer spectral formula is a direct relation, it can be differentiated for all allowed values of $\alpha$, including $\alpha=0$. This argument demonstrates that no extremals exist for logarithmic uncertainty.

Theorem 4. For $f \in \mathcal{S}\left(\mathbb{R}^{n}\right)$ and $g=|x|^{n / 2} f$

$$
\begin{aligned}
\int_{\mathbb{R}^{n}} \ln |x||f|^{2} d x+\int_{\mathbb{R}^{n}} \ln |\xi||\hat{f}|^{2} d \xi-D \int_{\mathbb{R}^{n}}|f|^{2} d x \\
\quad=\frac{1}{4} \pi^{-n / 2} \Gamma(n / 2) \int_{\mathbb{R}^{n} \times \mathbb{R}^{n}} \frac{|g(x)-g(y)|^{2}}{|x-y|^{n}}|x|^{-n / 2}|y|^{-n / 2} d x d y .
\end{aligned}
$$

with $D=\psi(n / 4)-\ln \pi$.

This result can be compared to the conformally invariant Hardy-Littlewood-Sobolev inequality for $L^{2}\left(\mathbb{R}^{n}\right)$ (see Theorem 3 in [6]):

Hardy-Littlewood-Sobolev inequality ( $L^{2}$ entropy form). For $f \in \mathcal{S}\left(\mathbb{R}^{n}\right)$ with $\|f\|_{2}=1$

$$
\begin{gathered}
\frac{n}{2} \int_{\mathbb{R}^{n}} \ln |\xi||\hat{f}(\xi)|^{2} d \xi \geq \int_{\mathbb{R}^{n}} \ln |f(x)||f(x)|^{2} d x+B_{n} \\
B_{n}=\frac{n}{2} \psi\left(\frac{n}{2}\right)-\frac{1}{2} \ln \left[\pi^{n / 2} \Gamma(n) / \Gamma(n / 2)\right]
\end{gathered}
$$

Up to conformal automorphism, extremal functions are of the form $A\left(1+|x|^{2}\right)^{-n / 2}$.

But observe that a weaker form of this inequality is given by "logarithmic uncertainty" (10) which will suffice by an asymptotic argument to determine the logarithmic Sobolev inequality in its Euclidean form (see [9], page 117).

Logarithmic Sobolev inequality. For $f \in \mathcal{S}\left(\mathbb{R}^{n}\right)$ with $\|f\|_{2}=1$

$$
\int_{\mathbb{R}^{n}}|f|^{2} \ln |f| d x \leq \frac{n}{4} \ln \left[\frac{2}{\pi e n} \int_{\mathbb{R}^{n}}|\nabla f|^{2} d x\right] .
$$

Theorem 5. Logarithmic uncertainly implies the logarithmic Sobolev inequality. 
Proof. Observe that for a non-negative radial decreasing function $h(x)$ with $\|h\|_{2}=1, h(x) \leq$ $m(B)^{-1 / 2}|x|^{-n / 2}$ where $m(B)$ is the volume of the unit ball. Then

$$
\int_{\mathbb{R}^{n}} \ln |x||h|^{2} d x \leq-\frac{2}{n} \int_{\mathbb{R}^{n}} \ln |h||h|^{2} d x-\frac{1}{n} \ln \left[\pi^{n / 2} / \Gamma\left(\frac{n}{2}+1\right)\right]
$$

and in general

$$
\int_{\mathbb{R}^{n}} \ln |\xi||\hat{h}|^{2} d \xi \leq \frac{1}{2} \ln \int_{\mathbb{R}^{n}}|\nabla h|^{2} d x-\ln (2 \pi)
$$

so that

$$
\begin{gathered}
\frac{1}{2} \ln \int_{\mathbb{R}^{n}}|\nabla h|^{2} d x-\frac{2}{n} \int_{\mathbb{R}^{n}} \ln |h||h|^{2} d x-\ln (2 \pi)-\frac{1}{n} \ln \left[\pi^{n / 2} / \Gamma\left(\frac{n}{2}+1\right)\right] \\
\geq \int_{\mathbb{R}^{n}} \ln |\xi||\hat{h}|^{2} d \xi+\int_{\mathbb{R}^{n}} \ln |x||h|^{2} d x \geq \psi(n / 4)-\ln \pi
\end{gathered}
$$

and

$$
\ln \int_{\mathbb{R}^{n}}|\nabla h|^{2} d x-\frac{4}{n} \int \ln |h||h|^{2} d x \geq 2 \psi(n / 4)+\ln (4 \pi)-\frac{2}{n} \ln \Gamma\left(\frac{n}{2}+1\right) .
$$

Since this result will hold for the equimeasurable radial decreasing rearrangement of a function and the left-hand side of this inequality decreases under symmetric rearrangement, it must hold for all functions $F$ with $\|F\|_{2}=1$ :

$$
\begin{aligned}
& \ln \left[\frac{2}{\pi e n} \int_{\mathbb{R}^{n}}|\nabla F|^{2} d x\right]-\frac{4}{n} \int_{\mathbb{R}^{n}} \ln |F||F|^{2} d x \\
& \geq 2 \psi(n / 4)+\ln (8 / e n)-\frac{2}{n} \ln \Gamma\left(\frac{n}{2}+1\right) .
\end{aligned}
$$

Set $n=k m$ with $F(x)=\prod f\left(x_{j}\right)$ for $j=1$ to $k$ with $x_{j} \in \mathbb{R}^{m}$ and $\|f\|_{2}=1$. Then (15) becomes

$$
\begin{gathered}
\ln \left[\frac{2}{\pi e m} \int_{\mathbb{R}^{m}}|\nabla f|^{2} d x\right]-\frac{4}{m} \int_{\mathbb{R}^{m}} \ln |f||f|^{2} d x \\
\geq 2 \psi\left(\frac{m k}{4}\right)+\ln \left[\frac{8}{e m k}\right]-\frac{2}{m k} \ln \Gamma\left(\frac{m k}{2}+1\right) \\
\simeq-\frac{1}{m k} \ln (\pi m k) \rightarrow 0
\end{gathered}
$$

as $k \rightarrow 0$. Hence

$$
\ln \left[\frac{2}{\pi e m} \int_{\mathbb{R}^{m}}|\nabla f|^{2} d x\right]-\frac{4}{m} \int_{\mathbb{R}^{m}} \ln |f||f|^{2} d x \geq 0
$$

and the proof of Theorem [5 is complete. A brief sketch of this argument was given in [9].

By utilizing the condition for equality in Theorem $1(\alpha=\beta)$, a second extension of logarithmic uncertainty is obtained through a differentiation argument.

Corollary to Theorem 1, For $f \in \mathcal{S}\left(\mathbb{R}^{n}\right)$ and $0 \leq \beta<2$

$$
\begin{aligned}
C_{\beta} \int_{\mathbb{R}^{n}}|\xi|^{\beta} \ln |\xi||\hat{f}|^{2} d \xi+\int_{\mathbb{R}^{n}}|x|^{-\beta} \ln |x||f|^{2} d x \\
\quad \geq\left(\frac{1}{2}\left[\psi\left(\frac{n+\beta}{4}\right)+\psi\left(\frac{n-\beta}{4}\right)\right]-\ln \pi\right) \int_{\mathbb{R}^{n}}|x|^{-\beta}|f|^{2} d x
\end{aligned}
$$




\section{Besov norms and the Hardy-Littlewood-Sobolev inequality.}

The nature of the weighted Besov norm can best be understood in terms of dilation invariance which allows its reformulation on the multiplicative group $\mathbb{R}_{+}$or the real line $\mathbb{R}$. Combine equation (12) with the $L^{2}$ Hardy-Littlewood-Sobolev inequality to obtain for $\|f\|_{2}=1$

$$
\begin{gathered}
\frac{1}{4} \pi^{-n / 2} \Gamma(n / 2) \int_{\mathbb{R}^{n} \times \mathbb{R}^{n}} \frac{|g(x)-g(y)|^{2}}{|x-y|^{n}}|x|^{-n / 2}|y|^{-n / 2} d x d y \\
\geq \frac{2}{n} \int_{\mathbb{R}^{n}} \ln |g||f|^{2} d x+E_{n} \\
E_{n}=\psi(n / 2)-\psi(n / 4)-\frac{1}{n} \ln \left[\pi^{-n / 2} \Gamma(n) / \Gamma(n / 2)\right] .
\end{gathered}
$$

Observe that due to equality in equation (12), inequality (17) is improved when $f$ is replaced by its equimeasurable radial decreasing rearrangement. Hence $f$ can be taken as radial and set $t=|x|, g(t)=g(x)$. Then

$$
\begin{gathered}
\frac{1}{4} \pi^{-n / 2} \Gamma(n / 2) \\
\int_{\mathbb{R}_{+} \times \mathbb{R}_{+}}|g(s)-g(t)|^{2} \varphi\left[\frac{s}{t}+\frac{t}{s}-2\right] \frac{d s}{s} \frac{d t}{t} \\
\geq \frac{2}{n} \int_{\mathbb{R}_{+}} \ln |g||g|^{2} \frac{d t}{t}+E_{n}
\end{gathered}
$$

and

Theorem 6. For $v \in \mathcal{S}(\mathbb{R})$ with $\|v\|_{2}=1$

$$
\begin{gathered}
\frac{1}{4} \pi^{-n / 2} \Gamma(n / 2) \int_{\mathbb{R} \times \mathbb{R}}|v(x)-v(y)|^{2} \varphi\left[4 \sinh ^{2}\left(\frac{x-y}{2}\right)\right] d x d y \\
\geq \frac{2}{n} \int_{\mathbb{R}} \ln |v||v|^{2} d x+E_{n} \\
\varphi(w)=\int_{S^{n-1}}\left[w+2\left(1-\xi_{1}\right)\right]^{-n / 2} d \xi
\end{gathered}
$$

where $d \xi$ is normalized surface measure. Extremal functions include $v(x)=A(\cosh x)^{-n / 2}$.

The full Hardy-Littlewood-Sobolev inequality can be used with the Frank-Lieb-Seiringer formula to give an embedding result on the multiplicative group in terms of the Besov norm.

Hardy-Littlewood-Sobolev inequality. For $f \in L^{p}\left(\mathbb{R}^{n}\right), 1<p<2$

$$
\begin{aligned}
& \left|\int_{\mathbb{R}^{n} \times \mathbb{R}^{n}} f(x)\right| x-\left.y\right|^{-2 n / p^{\prime}} g(y) d x d y \mid \leq A_{p}\|f\|_{L^{p}\left(\mathbb{R}^{n}\right)}\|g\|_{L^{p}\left(\mathbb{R}^{n}\right)} \\
& A_{p}=\pi^{n / p^{\prime}} \frac{\Gamma\left(\frac{n}{p}-\frac{n}{2}\right)}{\Gamma\left(\frac{n}{p}\right)}\left[\frac{\Gamma(n)}{\Gamma\left(\frac{n}{2}\right)}\right]^{2 / p-1} \\
& {\left[\|f\|_{L^{p^{\prime}}\left(\mathbb{R}^{n}\right)}\right]^{2} \leq C_{p} \int_{\mathbb{R}^{n}}\left|(-\Delta)^{n(2 / p-1) / 4} f\right|^{2} d x=(2 \pi)^{n(2 / p-1)} C_{p} \int_{\mathbb{R}^{n}}|\xi|^{n(2 / p-1)}|\hat{f}|^{2} d \xi} \\
& C_{p}=\frac{\Gamma\left(\frac{n}{p^{\prime}}\right)}{\Gamma\left(\frac{n}{p}\right)}\left[\frac{\Gamma(n)}{(4 \pi)^{n / 2} \Gamma\left(\frac{n}{2}\right)}\right]^{2 / p-1} .
\end{aligned}
$$


Set $\alpha=n(2 / p-1)$; then from (5) for $0<\alpha<\min \{2, n\}$ and $g=|x|^{(n-\alpha) / 2} f$

$$
\begin{aligned}
& C_{\alpha} \frac{\Gamma\left(\frac{n+\alpha}{2}\right)}{\Gamma\left(\frac{n-\alpha}{2}\right)}\left[\frac{\Gamma\left(\frac{n}{2}\right)}{\Gamma(n)}\right]^{\alpha / n}\left[\|f\|_{L^{p^{\prime}\left(\mathbb{R}^{n}\right)}}\right]^{2} \leq \int_{\mathbb{R}^{n}}|x|^{-\alpha}|f|^{2} d x \\
& +D_{\alpha} \int_{\mathbb{R}^{n} \times \mathbb{R}^{n}} \frac{|g(x)-g(y)|^{2}}{|x-y|^{n+\alpha}}|x|^{-(n-\alpha) / 2}|y|^{-(n-\alpha) / 2} d x d y .
\end{aligned}
$$

Note that for $n=2$ the condition on $\alpha$ allows the full range of values for $1<p<2$. Since $f$ can be taken to be radial, let $t=|x|$ and $f(x)=g(t) t^{-(n-\alpha) / 2}$; then one obtains the following Besov estimate on $\mathbb{R}_{+}$for $2<p^{\prime}<p_{c}$ where $p_{c}=2 n /(n-2)$ is the critical Sobolev embedding index on $\mathbb{R}^{n}$ for $n>2$.

Theorem 7. For $g \in L^{p^{\prime}}\left(\mathbb{R}_{+}\right)$with $\alpha=n\left(1-2 / p^{\prime}\right)$ and $n>2,2<p^{\prime}<2 n /(n-2)$

$$
\begin{aligned}
& C_{\alpha} \frac{\Gamma\left(\frac{n+\alpha}{2}\right)}{\Gamma\left(\frac{n-\alpha}{2}\right)}\left[\frac{\Gamma\left(\frac{n}{2}\right) \Gamma\left(\frac{n}{2}\right)}{2 \pi^{n / 2} \Gamma(n)}\right]^{\alpha / n}\|g\|_{L^{p^{\prime}\left(\mathbb{R}_{+}\right)}} \\
& \quad \leq\left[\|g\|_{L^{2}\left(\mathbb{R}_{+}\right)}\right]^{2}+D_{\alpha}\left[\frac{2 \pi^{n / 2}}{\Gamma\left(\frac{n}{2}\right)}\right] \int_{\mathbb{R}_{+} \times \mathbb{R}_{+}}|g(t)-g(s)|^{2} \psi_{\alpha, n}(t / s) \frac{d t}{t} \frac{d s}{s}
\end{aligned}
$$

where

$$
\psi_{\alpha, n}(t)=\int_{S^{n-1}}\left|t+\frac{1}{t}-2 \xi_{1}\right|^{-(n+\alpha) / 2} d \xi
$$

and

$$
\begin{gathered}
C_{\alpha}=\pi^{\alpha}\left[\Gamma\left(\frac{n-\alpha}{4}\right) / \Gamma\left(\frac{n+\alpha}{4}\right)\right]^{2}, \\
D_{\alpha}=\pi^{-n / 2-\alpha} \frac{\alpha}{4} \frac{\Gamma\left(\frac{n+\alpha}{2}\right)}{\Gamma\left(\frac{2-\alpha}{2}\right)} C_{\alpha} .
\end{gathered}
$$

The classical Aronszajn-Smith representation formula for the fractional Laplacian combined with the Hardy-Littlewood-Sobolev inequality allows one to obtain the sharp $L^{2}$ embedding constant for the Besov norm inequality considered by Bourgain, Brezis and Mironescu [16, 17, and Maz'ya and Shaposhnikova [29].

Theorem 8. For $F \in \mathcal{S}\left(\mathbb{R}^{n}\right), 0<\beta<1,2<q=2 n /(n-2 \beta)<p_{c}=2 n /(n-2)$

$$
\begin{aligned}
{\left[\|f\|_{L^{q}\left(\mathbb{R}^{n}\right)}\right]^{2} } & \leq C(n, 2, \beta) \int_{\mathbb{R}^{n} \times \mathbb{R}^{n}} \frac{|f(x)-f(y)|^{2}}{|x-y|^{n+2 \beta}} d x d y \\
C(n, 2, \beta) & =\frac{\beta(1-\beta)}{n-2 \beta} \pi^{-\beta-n / 2} \frac{\Gamma\left(\frac{n}{2}+1-\beta\right)}{\Gamma(2-\beta)}\left[\frac{\Gamma(n)}{\Gamma\left(\frac{n}{2}\right)}\right]^{2 \beta / n} .
\end{aligned}
$$

Proof. This result follows directly from the dual form of the Hardy-Littlewood-Sobolev inequality for the fractional Laplacian given by equation 21 (also see equation (18) in reference [9]) and the classical Aronszajn-Smith representation formula from the Appendix below (see (43)) by setting $p^{\prime}=q$ and $2 \beta=n(2 / p-1)$.

This inequality is sharp since for the allowed range of parameters it is equivalent to the HardyLittlewood-Sobolev inequality and one can calculate extremal functions using the extremals for that inequality. Observe that $C(n, 2, \beta)$ has the appropriate character determined by the 
respective principal theorems in Bourgain, Brezis and Mironescu (see equation (8), page 78 in [16]) and in Maz'ya and Shaposhnikova (see equation (2), page 231 in [29]).

\section{Besov norms and the nonlinear Stein-Weiss lemma.}

In the study and understanding of functional inequalities, symmetry often plays a central role, particularly in identifying optimal constants and extremal functions. A useful conceptual tool has been to transfer symmetry structures to analysis on a group (see [5] and [10]). The classical example is the Hardy-Littlewood paradigm that a positive integral operator that commutes with dilations may be reduced to Young's inequality for sharp convolution estimates on the multiplicative group. This principle is demonstrated here by giving short elementary proofs of two recent results by Frank and Seiringer [20, 21] on weighted inequalities and Besov norms to measure fractional smoothness. Symmetry invariance allows reduction of the problem to convolution estimates on a Lie group where a nonlinear Stein-Weiss lemma is used to obtain optimal estimates.

Theorem 9. Let $f \in \mathcal{S}\left(\mathbb{R}^{n}\right), 0<\beta<1$ and $1 \leq p<n / \beta$; then

$$
\begin{gathered}
\int_{\mathbb{R}^{n} \times \mathbb{R}^{n}} \frac{|f(x)-f(y)|^{p}}{|x-y|^{n+p \beta}} d x d y \geq D_{p, \beta} \int_{\mathbb{R}^{n}}|x|^{-p \beta}|f(x)|^{p} d x \\
D_{p, \beta}=\left.\left.\int_{\mathbb{R}^{n}}|1-| x\right|^{-\lambda}\right|^{p}|x-\eta|^{-n-p \beta} d x
\end{gathered}
$$

for $\lambda=(n-p \beta) / p$ and $\eta \in S^{n-1}$.

The proof depends on using the dilation invariance to transfer this inequality to a convolution problem on the multiplicative group and application of a new nonlinear Stein-Weiss lemma (see the appendix in [13] for the classical Stein-Weiss lemma).

Nonlinear Stein-Weiss Lemma. For $f, g \in L^{p}\left(\mathbb{R}^{m}\right), 1 \leq p<\infty$

$$
\begin{gathered}
\int_{\mathbb{R}^{m} \times \mathbb{R}^{m}}|g(y-x) f(x)-g(x-y) f(y)|^{p} d x d y \\
\geq \int_{\mathbb{R}^{m}}|| g(y)|-| g(-y)||^{p} d y \int_{\mathbb{R}^{m}}|f(x)|^{p} d x .
\end{gathered}
$$

Proof. By a change of variables in $y$ and using the triangle inequality for norms:

$$
\begin{aligned}
\int_{\mathbb{R}^{m} \times \mathbb{R}^{m}}|g(y-x) f(x)-g(x-y) f(y)|^{p} d x d y \\
\quad=\int_{\mathbb{R}^{m} \times \mathbb{R}^{m}}|g(y) f(x)-g(-y) f(x+y)|^{p} d x d y \\
\quad \geq \int_{\mathbb{R}^{m}}\left\{\left(\int_{\mathbb{R}^{m}}|g(y) f(x)-g(-y) f(x+y)|^{p} d x\right)^{1 / p}\right\}^{p} d y \\
\geq \int_{\mathbb{R}^{m}}\left\{|| g(y)\left|\|f\|_{L^{p}\left(\mathbb{R}^{n}\right)}-\right| g(-y)\left|\|f\|_{L^{p}\left(\mathbb{R}^{m}\right)}\right|\right\}^{p} d y \\
\quad=\int_{\mathbb{R}^{m}}|| g(y)|-| g(-y)||^{p} d y \int_{\mathbb{R}^{m}}|f(x)|^{p} d x .
\end{aligned}
$$

By considering $g \geq 0$ and the family $\varepsilon^{m / p} f(\varepsilon x)$, one observes that the inequality is optimal. 
On a Lie group $G$ with left-invariant Haar measure $d m$, modular function $\Delta$ and convolution defined by

then this lemma becomes:

$$
(f * g)(w)=\int_{G} f(z) g\left(z^{-1} w\right) d m
$$

Nonlinear Stein-Weiss Lemma on Groups. For $f, g \in L^{p}\left(\mathbb{R}^{n}\right), 1 \leq p<\infty$

$$
\begin{aligned}
\int_{G \times G} & \left|g\left(x^{-1} y\right) f(x)-g\left(y^{-1} x\right) f(y)\right|^{p} d m d m \\
& \geq \int_{G}|| g(y)\left|-\Delta(y)^{-1 / p}\right| g\left(y^{-1}\right)||^{p} d m \int_{G}|f(x)|^{p} d m .
\end{aligned}
$$

One recognizes that the argument is simply the interplay between the product structure and the Minkowski inequality for the $L^{p}$ metric.

Before beginning the proof of the main theorem, note that inequality (25) reduces to radial functions either by using the triangle inequality to effect the replacement

$$
f(x) \longrightarrow f_{\#}(|x|)=\left(\int_{S^{n-1}}|f(|x| \xi)|^{p} d \xi\right)^{1 / p}
$$

or use the symmetrization lemma from [3] to reduce the inequality to the equimeasurable radial decreasing rearrangement $f^{*}$, such that

$$
m\left\{x \in \mathbb{R}^{n}: f^{*}(x)>\lambda\right\}=m\left\{x \in \mathbb{R}^{n}:|f(x)|>\lambda\right\} .
$$

Symmetrization Lemma. The functional

$$
\Lambda(f, g)=\int_{M \times M} \varphi\left[\frac{|f(x)-g(y)|}{\rho(d(x, y))}\right] \kappa[d(x, y)] d x d y
$$

is monotone under equimeasurable radial decreasing rearrangement: $\Lambda(f, g) \geq \Lambda\left(f^{*}, g^{*}\right)$. $M$ is a manifold with distance function $d(x, y)$ and reflection symmetry, and $\varphi, \rho, \kappa$ are non-negative functions on $[0, \infty)$ with the properties: (i) $\varphi(0)=0, \varphi$ convex and monotone increasing, and $t \varphi^{\prime}(t)$ convex; (ii) $\rho$ monotone increasing, $\kappa$ monotone decreasing.

Proof of Theorem. Assume that $f$ is now radial; set $t=|x|, s=|y|, h(t)=|x|^{\frac{n}{p}-\beta} f(x)$. Then inequality (25) is inequivalent to the following form on the multiplicative group $\mathbb{R}_{+}$.

$$
\int_{\mathbb{R}_{+} \times \mathbb{R}_{+}}|g(s / t) h(t)-g(t / s) h(s)|^{p} \psi(s / t) \frac{d s}{s} \frac{d t}{t} \geq D_{p, \beta} \int_{\mathbb{R}_{+}}|h(t)|^{p} \frac{d t}{t}
$$

where $g(t)=t^{(n-p \beta) / 2 p}$,

$$
\psi(t)=\int_{S^{n-1}}\left[t+\frac{1}{t}-25_{1}\right]^{-(n+p \beta) / 2} d \xi
$$

and $d \xi$ denotes standard surface measure on $S^{n-1}$. Note that $\psi$ is symmetric under inversion. Apply the nonlinear Stein-Weiss lemma using $g \psi^{1 / p}$ as the second function in the Lemma, and one finds that

$$
\begin{aligned}
D_{p, \beta} & =\int_{\mathbb{R}_{+}}\left|t^{\lambda / 2}-t^{-\lambda / 2}\right|^{p} \psi(t) \frac{d t}{t} \\
& =\left.\left.\int_{\mathbb{R}^{n}}|1-| x\right|^{-\lambda}\right|^{p}|x-\eta|^{-n-p \beta} d x
\end{aligned}
$$


where $\lambda=(n-p \beta) / p$ and $\eta \in S^{n-1}$. Since the determination of $D_{p, \beta}$ depends only on application of the Stein-Weiss lemma, the constant must be optimal as observed by a suitable variation of functions in that inequality.

In contrast to the original Stein-Weiss Lemma (see appendix in [13, the key step in the proof of the nonlinear form is identified here as the "lemma". This is largely due to the surprising simplicity and the form of the argument plus its natural extension to a non-unimodular Lie groups. But for completeness Theorem 9 is reformulated to have the "look" of the original Stein-Weiss lemma with the same proof as above.

Stein-Weiss Lemma (extended). Suppose $K$ is a non-negative symmetric kernel defined on $\mathbb{R}^{n} \times \mathbb{R}^{n}$, continuous on any domain that excludes the diagonal, homogeneous of degree $-n-\gamma$, $K(\delta u, \delta v)=\delta^{-n-\gamma} K(u, v), 0<\gamma<\min (n, p)$, and $K(R u, R v)=K(u, v)$ for any $R \in S O(n)$. Then for $f \in \mathcal{S}\left(\mathbb{R}^{n}\right)$ and $p \geq 1$,

$$
\begin{gathered}
\int_{\mathbb{R}^{n} \times \mathbb{R}^{n}}|f(x)-f(y)|^{p} K(x, y) d x d y \geq D_{p, \gamma} \int_{\mathbb{R}^{n}}|x|^{-\gamma}|f(x)|^{p} d x \\
D_{p, \gamma}=\left.\left.\int_{\mathbb{R}^{n}}|1-| x\right|^{-\lambda}\right|^{p} K(x, \eta) d x
\end{gathered}
$$

for $\lambda=(n-\gamma) / p$ and $\eta \in S^{n-1}$. This constant is optimal. But note that there is no assumption made that it is finite.

In the context of Maz'ya-Sobolev embedding, Frank and Seiringer [21] have given an extension of Theorem 9 to the setting of the upper half-space $\mathbb{R}_{+}^{n}$ using a similar argument to their earlier proof of Theorem 9 [20]. But the nonlinear Stein-Weiss lemma for non-unimodular groups can also be used to give a simple proof. Note that for both of these inequalities this approach does not give the error estimates obtained by Frank and Seiringer. To set notation, let $w=(x, y) \in \mathbb{R}_{+}^{n}$ with $x \in \mathbb{R}^{n-1}, y>0$.

Theorem 10. For $f \in \mathcal{S}\left(\mathbb{R}_{+}^{n}\right), 0<\beta<1$ and $1 \leq p<1 / \beta$

$$
\begin{gathered}
\int_{\mathbb{R}_{+}^{n} \times \mathbb{R}_{+}^{n}} \frac{\left|f(w)-f\left(w^{\prime}\right)\right|^{p}}{\left|w-w^{\prime}\right|^{n+p \beta}} d w d w^{\prime} \geq E_{p, \beta} \int_{\mathbb{R}_{+}^{n}}|f(w)|^{p} y^{-p \beta} d w \\
E_{p, \beta}=\frac{\pi^{\frac{n-1}{2}} \Gamma\left(\frac{p \beta+1}{2}\right)}{\Gamma\left(\frac{p \beta+n}{2}\right)} \int_{0}^{\infty}\left|1-y^{\beta-\frac{1}{p}}\right|^{p}|y-1|^{-1-p \beta} d y .
\end{gathered}
$$

This constant is optimal.

Proof. The basic step is to convert this inequality to a problem on $n$-dimensional hyperbolic space $\mathbb{H}^{n}$ and apply the nonlinear Stein-Weiss lemma for groups. Here the Liouville-Beltrami upper half-space model is used with: Poincaré distance

$$
d\left(w, w^{\prime}\right)=\frac{\left|w-w^{\prime}\right|}{2 \sqrt{y y^{\prime}}}
$$

left-invariant Haar measure $d \nu=y^{-n} d x d y$, Riemannian metric $d s^{2}=y^{-2}\left(d x^{2}+d y^{2}\right)$ and invariant gradient $D=y \nabla$. The group structure of hyperbolic space corresponds to a nonunimodular Lie group that is an extension of the affine " $a x+b$ group." Hyperbolic space $\mathbb{H}^{n}$ is identified with the subgroup of $S L(n, R)$ given by matrices of the form

$$
\sqrt[n]{y}\left(\begin{array}{cc}
\mathbf{1} & x / y \\
0 & 1 / y
\end{array}\right)
$$


where $x \in \mathbb{R}^{n-1}$ is represented as a column vector and $y>0$. Such matrices can act via fractional linear transformation on $\mathbb{R}_{+}^{n} \simeq \mathbb{H}^{n}$

$$
w=x+i y \xi \in \mathbb{R}_{+}^{n} \longrightarrow \frac{A w+B}{C w+D}
$$

for a matrix $\left(\begin{array}{cc}A & B \\ C & D\end{array}\right)$ where $A=(n-1) \times(n-1)$ matrix, $B=(n-1) \times 1$ matrix, $C=1 \times(n-1)$ matrix, $D=1 \times 1$ matrix and fixed non-zero $\xi \in \mathbb{R}^{n-1}$. The group action then corresponds to the multiplication rule

$$
(x, y)(u, v)=(x+y u, y v)
$$

for $x, u \in \mathbb{R}^{n-1}$ and $y, v>0$ so this is a non-unimodular group. Let $\Delta$ denote the modular function defined by $\nu(E g)=\Delta(g) \nu(E)$. Then $\Delta(x, y)=y^{-(n-1)}$.

Returning to equation (29), let $F(w)=f(w) y^{\lambda}$ with $\lambda=\frac{n}{p}-\beta$. Then this equation becomes

$$
\begin{gathered}
2^{-n-p \beta} \int_{\mathbb{H}^{n} \times \mathbb{H}^{n}} \frac{\left|F(w)\left(y^{\prime} / y\right)^{\lambda / 2}-F\left(w^{\prime}\right)\left(y / y^{\prime}\right)^{\lambda / 2}\right|^{p}}{d\left(w, w^{\prime}\right)^{n+p \beta}} d \nu d \nu= \\
2^{-n-p \beta} \int_{\mathbb{H}^{n} \times \mathbb{H}^{n}}\left|g\left(w^{-1} w^{\prime}\right) F(w)-g\left(w^{\prime-1} w\right) F\left(w^{\prime}\right)\right|^{p} d \nu d \nu \geq E_{p, \beta} \int_{\mathbb{H}^{n}}|F|^{p} d \nu
\end{gathered}
$$

where $g(w)=y^{\lambda / 2} d(w, \hat{0})^{-\left(\frac{n}{p}+\beta\right)}$ with $\hat{0}=(0,1)$. Then applying the nonlinear Stein-Weiss lemma for non-unimodular groups

$$
\begin{aligned}
E_{p, \beta} & =\int_{\mathbb{H}^{n}}\left|y^{\lambda / 2}-y^{(n-1) / p} y^{-\lambda / 2}\right|^{p} y^{(n+p \beta) / 2}\left(x^{2}+(y-1)^{2}\right)^{-(n+p \beta) / 2} d \nu \\
& =\frac{\pi^{\frac{n-1}{2}} \Gamma\left(\frac{p \beta+1}{2}\right)}{\Gamma\left(\frac{p \beta+n}{2}\right)} \int_{0}^{\infty}\left|1-y^{\beta-\frac{1}{p}}\right|^{p}|y-1|^{-1-p \beta} d y
\end{aligned}
$$

Note that no information results if the case $p \beta=1$ is treated as a limiting case.

Alternate proof. The interplay between the product structure of the manifold and the "triangle inequality" for $L^{p}$ norms offers a simpler proof as a direct application of Theorem 9. (To better understand this variation in proof, see the discussion on page 832 in [8].)

$$
\begin{aligned}
\int_{\mathbb{R}_{+}^{n} \times \mathbb{R}_{+}^{n}} & \frac{\left|f(w)-f\left(w^{\prime}\right)\right|^{p}}{\left|w-w^{\prime}\right|^{n+p \beta}} d w d w^{\prime} \geq \int_{\mathbb{R}_{+} \times \mathbb{R}_{+}}\left|h(y)-h\left(y^{\prime}\right)\right|^{p} J\left(y-y^{\prime}\right) d y d y^{\prime} \\
\quad= & \left.\left.\int_{\mathbb{R}^{n-1}}|1+| x\right|^{2}\right|^{-n-p \beta} d x \int_{\mathbb{R}_{+} \times \mathbb{R}_{+}}\left|h(y)-h\left(y^{\prime}\right)\right|^{p}\left|y-y^{\prime}\right|^{-1-p \beta} d y d y^{\prime} \\
\geq & \frac{\pi^{\frac{n-1}{2}} \Gamma\left(\frac{p \beta+1}{2}\right)}{\Gamma\left(\frac{p \beta+h}{2}\right)} \int_{0}^{\infty}\left|1-y^{\beta-\frac{1}{p}}\right|^{p}|y-1|^{-1-p \beta} d y \int_{0}^{\infty}|h(y)|^{p} y^{-p \beta} d y \\
= & \pi^{\frac{n-1}{2}} \frac{\Gamma\left(\frac{p \beta+1}{2}\right)}{\Gamma\left(\frac{p \beta+h}{2}\right)} \int_{0}^{\infty}\left|1-y^{\beta-\frac{1}{p}}\right|^{p}|y-1|^{-1-p \beta} d y \int_{\mathbb{R}_{+}^{n}}|f(w)|^{p} y^{-p \beta} d w
\end{aligned}
$$

where

$$
h(y)=\left(\int_{\mathbb{R}^{n-1}}|f(x, y)|^{p} d x\right)^{1 / p}, \quad J(v)=\int_{\mathbb{R}^{n-1}}\left|x^{2}+v^{2}\right|^{-n-p \beta} d x .
$$

This argument suggests an immediate application to two additional cases: the Heisenberg group and manifolds with mixed homogeneity. Geometric analysis on the Heisenberg group depends in part on understanding the intrinsic $S L(2, R)$ invariance associated with the group 
manifold. But in studying manifolds where homogeneity is "broken", it is natural to determine if the Besov norm estimates obtained for $\mathbb{R}^{n}$ and the upper half-space $\mathbb{R}_{+}^{n}$ can extend simply to the Heisenberg group. In the context of Stein-Weiss integrals, a similar question was considered earlier in [8].

The Heisenberg group $\mathcal{H}_{n}$ is realized as the boundary of the Siegel upper half-space in $\mathbb{C}^{n+1}$, $D=\left\{z \in \mathbb{C}^{n+1}: \operatorname{Im} z_{n+1}>\left|z_{1}\right|^{2}+\cdots+\left|z_{n}\right|^{2}\right\}$. Then $\mathcal{H}_{n}=\left\{w=(z, t): z \in \mathbb{C}^{n}, t \in \mathbb{R}\right\}$ with the group action

$$
w w^{\prime}=(z, t)\left(z^{\prime}, t^{\prime}\right)=\left(z+z^{\prime}, t+t^{\prime}+2 \operatorname{Im} z \bar{z}^{\prime}\right)
$$

and Haar measure on the group is given by $d w=d z d \bar{z} d t=4^{n} d x d y d t$ where $z=z+i y \in \mathbb{C}^{n}$ and $t \in \mathbb{R}$. The natural metric here is $d\left(w, w^{\prime}\right)=d\left((z, t),\left(z^{\prime}, t^{\prime}\right)\right)=d\left(w^{\prime}-1 w, \hat{0}\right)$ with

$$
d(w, \hat{0})=d((z, t), 0,0))=\left.|| z\right|^{2}+\left.i t\right|^{1 / 2}=\left.|| z\right|^{4}+\left.t^{2}\right|^{1 / 4} .
$$

Theorem 11 (Besov norms on the Heisenberg group). Let $f \in \mathcal{S}\left(\mathcal{H}_{n}\right), 0<\beta<1$ and $1 \leq p<2 n / \beta ;$ then

$$
\begin{gathered}
\int_{\mathcal{H}_{n} \times \mathcal{H}_{n}} \frac{\left|f(w)-f\left(w^{\prime}\right)\right|^{p}}{d\left(w, w^{\prime}\right)^{2 n+2+p \beta}} d w d w^{\prime} \geq F_{p, \beta} \int_{\mathcal{H}_{n}}|z|^{-p \beta}|f|^{p} d w \\
F_{p, \beta}=\left.\left.\frac{4^{n} \sqrt{\pi} \Gamma\left[\frac{2 n+p \beta}{4}\right]}{\Gamma\left[\frac{2 n+2+p \beta}{4}\right]} \int_{\mathbb{R}^{n}}|1-| x\right|^{-\lambda}\right|^{p}|x-\eta|^{-2 n-p \beta} d x
\end{gathered}
$$

for $\lambda=(2 n-p \beta) / p$ and $\eta \in S^{2 n-1}$.

Proof. Apply the "triangle inequality" to the $t, t^{\prime}$ integrations

$$
\begin{aligned}
\int_{\mathcal{H}_{n} \times \mathcal{H}_{n}} & \frac{\left|f(w)-f\left(w^{\prime}\right)\right|^{p}}{d\left(w, w^{\prime}\right)^{2 n+2+p \beta}} d w d w^{\prime} \\
\geq & \int_{\mathbb{C}^{n} \times \mathbb{C}^{n}}\left|h(z)-h\left(z^{\prime}\right)\right|^{p} \int_{\mathbb{R}}\left[\left|z-z^{\prime}\right|^{4}+t^{2}\right]^{-(2 n+2+p \beta) / 4} d t d z d \bar{z} d z^{\prime} d \bar{z}^{\prime} \\
= & \frac{\sqrt{\pi} \Gamma\left[\frac{2 n+p \beta}{4}\right]}{\Gamma\left[\frac{2 n+2+p \beta}{4}\right]} \int_{\mathbb{C}^{n} \times \mathbb{C}^{n}} \frac{\left|h(z)-h\left(z^{\prime}\right)\right|^{p}}{\left|z-z^{\prime}\right|^{2 n+p \beta}} d z d \bar{z} d z^{\prime} d \bar{z}^{\prime} \\
\geq & \left.\left.\frac{4^{n} \sqrt{\pi} \Gamma\left[\frac{2 n+p \beta}{4}\right]}{\Gamma\left[\frac{2 n+2+p \beta}{4}\right]} \int_{\mathbb{R}^{2 n}}|1-| x\right|^{-\lambda}\right|^{p}|x-\eta|^{-2 n-p \beta} d x \int_{\mathbb{C}^{n}}|z|^{-p \beta}|h|^{p} d x d \bar{z}
\end{aligned}
$$

for $\lambda=(2 n-p \beta) / p$ and $\eta \in S^{2 n-1}$ where Theorem 9 is applied to obtain this estimate with

$$
h(z)=\left(\int_{\mathbb{R}}|f(z, t)|^{p} d t\right)^{1 / p}
$$

so that inequality 30 is obtained.

A similar result holds for problems with mixed homogeneity (see the corresponding discussion for Stein-Weiss potentials on page 1876 in [13]). 
Theorem 12 (Besov norms with mixed homogeneity). Let $f \in \mathcal{S}\left(\mathbb{R}^{n+m}\right), 0<\beta<1$ and $1 \leq p<m / \beta, w=(x, v) \in \mathbb{R}^{n} \times \mathbb{R}^{m} ;$ then

$$
\begin{gathered}
\int_{\mathbb{R}^{n+m} \times \mathbb{R}^{n+m}} \frac{\left|f(w)-f\left(w^{\prime}\right)\right|}{\left|w-w^{\prime}\right|^{n+m+p \beta}} d w d w^{\prime} \geq G_{p, \beta} \int_{\mathbb{R}^{n} \times \mathbb{R}^{m}}|v|^{-p \beta}|f|^{p} d w \\
G_{p, \beta}=\left.\left.\frac{\pi^{n / 2} \Gamma\left[\frac{m+p \beta}{2}\right]}{\Gamma\left[\frac{n+m+p \beta}{2}\right]} \int_{\mathbb{R}^{m}}|1-| x\right|^{-\lambda}\right|^{p}|x-\eta|^{-m-p \beta} d x
\end{gathered}
$$

for $\lambda=(m-p \beta) / p$ and $\eta \in S^{m-1}$.

Proof. Apply the previous argument using the "triangle inequality" for the $x, x^{\prime}$ integration and Theorem 9, Observe that $G_{p, \beta}$ is then given by

$$
\left.\left.\int_{\mathbb{R}^{n}}\left[1+|x|^{2}\right]^{-(n+m+p \beta) / 2} d x \int_{\mathbb{R}^{m}}|1-| x\right|^{-\lambda}\right|^{p}|x-\eta|^{-m-p \beta} d x
$$

with $\lambda, \eta$ as above.

\section{Analysis and applications.}

The Frank-Lieb-Seiringer spectral formula which relates the $L^{2}$ norm for the fractional Laplacian to the weighted Besov norm can be applied in different ways to give sharp information on embedding questions. First, it can be used to obtain global error estimates for Hardy's inequality and Pitt's inequality that simultaneously demonstrate sharpness and that optimal constants are not attained. But more generally, this formula can be combined with the HardyLittlewood-Sobolev inequality to obtain or recover sharp embedding constants. Results from [7] can be viewed as predictive for this conceptual framework. To illustrate this strategy, the Hardy relation on $\mathbb{R}^{3}$.

$$
\int_{\mathbb{R}^{3}}|\nabla f|^{2} d x=\frac{1}{4} \int_{\mathbb{R}^{3}}|x|^{-2}|f|^{2} d x+\int_{\mathbb{R}^{3}}\left|\nabla\left(|x|^{1 / 2} f\right)\right|^{2}|x|^{-1} d x
$$

can be used to obtain the sharp $H^{1}(\mathbb{R})$ Moser inequality calculated by Nagy [30] (see also [11])

$$
\int_{\mathbb{R}}|g|^{6} d x \leq \frac{4}{\pi^{2}} \int_{\mathbb{R}}|\nabla g|^{2} d x\left[\int_{\mathbb{R}}|g|^{2} d x\right]^{2} .
$$

The Sobolev inequality on $\mathbb{R}^{3}$ is

$$
3(\pi / 2)^{4 / 3}\left(\int_{\mathbb{R}^{3}}|f|^{6} d x\right)^{1 / 3} \leq \int_{\mathbb{R}^{3}}|\nabla f|^{2} d x
$$

which results using (6)

$$
3(\pi / 2)^{4 / 3}\left(\int_{\mathbb{R}^{3}}|f|^{6} d x\right)^{1 / 3} \leq \frac{1}{4} \int_{\mathbb{R}^{3}}|x|^{-2}|f|^{2} d x+\int_{\mathbb{R}^{3}}\left|\nabla\left(|x|^{1 / 2} f\right)\right|^{2}|x|^{-1} d x .
$$

Choose $f$ to be radial, set $t=|x|$ and $h(t)=|x|^{1 / 2} f(x)$; then let $t=e^{w}, g(w)=h\left(e^{w}\right)$ and apply a variational argument to obtain (32)

$$
\int_{\mathbb{R}}|g|^{6} d w \leq \frac{4}{\pi^{2}} \int_{\mathbb{R}}|\nabla g|^{2} d w\left[\int_{\mathbb{R}}|g|^{2} d w\right]^{2} .
$$

Though the context here is the real line, differentiation is denoted by the gradient symbol $\nabla$ to reflect the character of embedding relations. Using the Hardy-Littlewood-Sobolev inequality, 
the optimal constant is obtained for the extremal function $(\cosh w)^{-1 / 2}$. A different characterization of this approach would be that it provides a clever method to discover useful change of variables that develop more fully the underlying symmetry of embedding estimates.

This argument can be used with sharp Sobolev embedding on $\mathbb{R}^{n}$ for $n>2, p^{\prime}=2 n /(n-2)$ or $n / p^{\prime}=n / 2-1$

$$
\pi n(n-2)[\Gamma(n / 2) / \Gamma(n)]^{2 / n}\left(\int_{\mathbb{R}^{n}}|f|^{p^{\prime}} d x\right)^{2 / p^{\prime}} \leq \int_{\mathbb{R}^{n}}|\nabla f|^{2} d x
$$

which gives by setting $s=n / p^{\prime}=n / 2-1$ and using (6)

$$
\begin{gathered}
4 \pi s(s+1)[\Gamma(s+1) / \Gamma(2 s+2)]^{1 /(s+1)}\left(\int_{\mathbb{R}^{n}}|f|^{2+2 / s} d x\right)^{s /(s+1)} \\
\leq s^{2} \int_{\mathbb{R}^{n}}|x|^{-2}|f|^{2} d x+\int_{\mathbb{R}^{n}}\left|\nabla\left(|x|^{2} f\right)\right|^{2}|x|^{-2 s} d x .
\end{gathered}
$$

Choose $f$ to be radial, set $t=|x|$ and $h(t)=|x|^{s} f(x)$; then

$$
\begin{aligned}
& 2^{(2 s+1) /(s+1)} s(s+1) {\left[\Gamma^{2}(s+1) / \Gamma(2 s+2)\right]^{1 /(s+1)}\left(\int_{0}^{\infty}|h|^{2+2 / s} \frac{d t}{t}\right)^{s /(s+1)} } \\
& \leq s^{2} \int_{0}^{\infty}|h|^{2} \frac{d t}{t}+\int_{0}^{\infty}\left|t h^{\prime}\right|^{2} \frac{d t}{t}
\end{aligned}
$$

now let $t=e^{w}$ and $g(w)=h\left(e^{w}\right)$ to obtain an $H^{\prime}$ Sobolev inequality on the real line

$$
\begin{aligned}
& A_{s}\left(\int_{\mathbb{R}}|g|^{2+2 / s} d w\right)^{s /(s+1)} \leq s^{2} \int_{\mathbb{R}}|g|^{2} d w+\int_{\mathbb{R}}|\nabla g|^{2} d w \\
& A_{s}=2^{(2 s+1) /(s+1)} s(s+1)\left[\Gamma^{2}(s+1) / \Gamma(2 s+2)\right]^{1 /(s+1)} .
\end{aligned}
$$

Using a standard variational argument $\{g(w) \rightarrow g(\delta w), \delta>0\}$, one finds the equivalent Gagliardo-Nirenberg inequality which was originally calculated by Nagy [30].

Theorem 13. For $g \in C^{1}(\mathbb{R})$ and $0<s<\infty$

$$
\begin{gathered}
\left(\int_{\mathbb{R}}|g|^{2+2 / s} d x\right)^{2 s} \leq B_{s} \int_{\mathbb{R}}|\nabla g|^{2} d x\left(\int_{\mathbb{R}}|g|^{2} d x\right)^{2 s+1} \\
B_{s}=(s / 2)^{2 s}(2 s+1)^{-(2 s+1)} \frac{\Gamma^{2}(2 s+2)}{\Gamma^{4}(s+1)} .
\end{gathered}
$$

An extremal function is given by $(\cosh x)^{-s}$.

Proof. For the case where $s$ is a half-integer multiple, this inequality follows the previous deduction using sharp Sobolev embedding on $\mathbb{R}^{n}$. The extremal function follows from the analysis on $\mathbb{R}^{n}$. But the general case depends only on observing that an extremal function exists by duality for the $H^{1}$ inequality, and that one can find a unique radial-decreasing solution for the Euler-Lagrange variational equation. The argument is simple and follows the outline of the Hardy-Littlewood-Sobolev method used in [10] (see page 1244). The two inequalities for 
$1 / p+1 / q=1,2<q<q_{c}=2 n /(n-2)$ for $n>2, q_{c}=\infty$ if $n=1,2$

$$
\begin{gathered}
\left(\int_{\mathbb{R}^{n}}|g|^{q} d x\right)^{2 / q} \leq A_{q}\left[\int_{\mathbb{R}^{n}}|g|^{2} d x+\int_{\mathbb{R}^{n}}|\nabla g|^{2} d x\right] \\
\left|\int_{\mathbb{R}^{n} \times \mathbb{R}^{n}} f(x) G_{2}(x-y) f(y) d x d y\right| \leq A_{q}\left[\|f\|_{L^{p}\left(\mathbb{R}^{n}\right)}\right]^{2}
\end{gathered}
$$

are equivalent by duality and the existence of an extremal function for (37) will imply existence of an extremal function for (36), in particular $g=G_{2} * f$ where $G_{2}$ is the Bessel potential defined using the Fourier transform by

$$
\hat{G}_{\alpha}(\xi)=\left(1+4 \pi^{2}|\xi|^{2}\right)^{-\alpha / 2}
$$

with

$$
\begin{aligned}
G_{\alpha}(x) & =\left[(4 \pi)^{\alpha / 2} \Gamma(\alpha / 2)\right]^{-1} \int_{0}^{\infty} e^{-\pi|x|^{2} / \delta} e^{-\delta / 4 \pi} \delta^{(-n+\alpha) / 2} \frac{d \delta}{\delta} \\
& =\left[2^{(n+\alpha-2) / 2} \pi^{n / 2} \Gamma(\alpha / 2)\right]^{-1}|x|^{-(n-\alpha) / 2} K_{(n-\alpha) / 2}(|x|) .
\end{aligned}
$$

Note that $G_{\alpha}(x)$ is radial decreasing. Since $\hat{G}_{\alpha}$ is positive, the convolution operator defined by $G_{\alpha}$ is positive-definite and it suffices to study its properties on the diagonal. By symmetrization, it suffices to treat (36) for non-negative radial decreasing functions. To show the existence of extremal functions, consider a sequence of non-negative radial decreasing functions $\left\{f_{m}\right\}$ with $\left\|f_{m}\right\|_{p}=1$ and

$$
\int_{\mathbb{R}^{n} \times \mathbb{R}^{n}} f_{m}(x) G_{2}(x-y) f_{m}(y) d x d y \underset{m \rightarrow \infty}{\longrightarrow} A_{q} .
$$

By virtue of the norm condition, $f_{m}(x) \leq c|x|^{-n / p}$. Using the Helly selection principle, one can choose a subsequence that converges almost everywhere to a function $F$. By Fatou's lemma, $\|F\|_{p} \leq 1$. But

$$
f_{m}(x) G_{2}(x-y) f_{m}(y) \leq c^{2}|x|^{-n / p} G_{2}(x-y)|y|^{-n / p} \in L^{1}\left(\mathbb{R}^{n} \times \mathbb{R}^{n}\right)
$$

for $2<q<q_{c}$ where $q$ is the dual exponent to $p$. Using the Fourier transform

$$
\int_{\mathbb{R}^{n} \times \mathbb{R}^{n}}|x|^{-n / p} G_{2}(x-y)|y|^{-n / p} d x d y=c \int_{\mathbb{R}^{n}}|\xi|^{-2 n / q}\left(1+4 \pi^{2}|\xi|^{2}\right)^{-1} d \xi<\infty
$$

for $2<q<q_{c}$. This is a nice calculation because it highlights the role of the critical index to ensure that the right-hand integral is finite. Now using the dominated convergence theorem, the existence of an extremal function is demonstrated

$$
\int_{\mathbb{R}^{n} \times \mathbb{R}^{n}} f_{m}(x) G_{2}(x-y) f_{m}(y) d x d y \rightarrow \int_{\mathbb{R}^{n} \times \mathbb{R}^{n}} F(x) G_{2}(x-y) F(y) d x d y=A_{q}
$$

with $\|F\|_{p}=1$. The existence of an extremal function for (36) means that an extremal function will exist for the $H^{1}$ Sobolev embedding estimate (36), and in fact one can take $g=G_{2} * F$ which is bounded and radial decreasing since $G_{2} \in L^{r}\left(\mathbb{R}^{n}\right)$ for $1 \leq r<q_{c}$. Now this extremal function will be a non-negative bounded radial decreasing solution of the Euler-Lagrange variational equation

$$
-\Delta g+g=c g^{q-1}
$$

For the case $n=1$ where by rescaling the equation becomes

$$
-g^{\prime \prime}+s^{2} g=c g^{1+2 / s}
$$


which has a unique bounded symmetric decreasing solution, $g(x)=(\cosh x)^{-s}$. The proof of Theorem 13 is completed by using the extremal function to compute the constant in (37) and then applying the variational argument to obtain (35). This is the only case in which an explicit closed-form solution for the extremal function and the constant $A_{q}$ has been calculated (see Nagy [30]). Weinstein [41] calculated numerically the value of $A_{4}$ in dimension two and suggested that his methods could be applied to calculate any of the other constants. But relatively simple approximations can be used to obtain good numerical values (see [11], pages 355-357). The $n$-dimensional results detailed above are expressed in the following theorem.

Theorem 14. For $f \in L^{p}\left(\mathbb{R}^{n}\right), g \in C^{1}\left(\mathbb{R}^{n}\right) \cap L^{2}\left(\mathbb{R}^{n}\right)$ with $1 / p+1 / q=1,2<q<q_{c}=2 n /(n-2)$ for $n>2, q_{c}=0 \infty$ if $n=1$ or 2 , the following inequalities are equivalent

$$
\begin{gathered}
\left|\int_{\mathbb{R}^{n} \times \mathbb{R}^{n}} f(x) G_{2}(x-y) f(y) d x d y\right| \leq A_{q}\left[\|f\|_{L^{p}\left(\mathbb{R}^{n}\right)}\right]^{2} \\
{\left[\|g\|_{L^{q}\left(\mathbb{R}^{n}\right)}\right]^{2} \leq A_{q}\left[\int_{\mathbb{R}^{n}}|\nabla g|^{2} d x+\int_{\mathbb{R}^{n}}|g|^{2} d x\right] .}
\end{gathered}
$$

Here

$$
\hat{G}_{2}(\xi)=\left(1+4 \pi^{2}|\xi|^{2}\right)^{-1} .
$$

Bounded positive radial decreasing extremal functions exist for both inequalities. For the $H^{1}$ Sobolev inequality, the extremal will be a radial solution of the differential equation

$$
-\Delta g+g=c g^{q-1} .
$$

A further interesting remark can be made for the one-dimensional setting by using equation (34). Set

$$
d \nu=(\cosh w)^{-2 \delta-2} d w, \quad D=\cosh w \frac{d}{d w}
$$

and $g=(\cosh w)^{-s} k$. Then equation (34) becomes

$$
A_{s}\left[\|k\|_{L^{2+2 / s}(d \nu)}\right]^{2} \leq \int|D k|^{2} d \nu+s(s+1) \int|k|^{2} d \nu
$$

which now has some resemblance to Sobolev embedding estimates on a curved manifold.

Remarks. Pitt formulated inequality (2) for Fourier series. Zygmund viewed this result as generalizing theorems of Hardy-Littlewood and Paley for weighted norms of Fourier coefficients (see notes for chapter 12 in Trigonometric Series). Extensions of Pitt's inequality for Fourier coefficients of uniformly bounded orthonormal systems are given in [34], [38. Clearly Pitt's inequality encompasses the Hausdorff-Young inequality as well as the conformally invariant Hardy-Littlewood-Sobolev inequality. A proof for the Euclidean $\mathbb{R}^{n}$ version of Pitt's inequality is given in the Appendix to the author's paper [13. Calculation of the best constant for the $L^{2}$ inequality (3) was done independently by Herbst [24], Yafaev [42] and the author [6]. In the recent literature, this inequality has been characterized as a Hardy-Rellich inequality or a Hardy-type inequality following the case $\alpha=2$. The optimal constant for the Stein-Weiss inequality (4) was obtained independently by Samko [33] and the author [13]. The "ground state spectral representation" (5) appears in the recent paper by Frank, Lieb and Seiringer [19]. A different proof is given in the appendix below. The proofs given for the calculation of optimal constants for diagonal maps have some overall similarity, but the arguments given by the author (6], 8], [12, [13]) emphasize the geometric symmetry corresponding to dilation invariance and characterize the operators in terms of convolution, as does the proof by Herbst 24]. The proofs given in [26] reflect more the simplicity of Schur's lemma, but an independent argument must be given to show that the bound is optimal. The method used by Samko [33] (see Theorem 6.4, 
page 70 in [25]) is to essentially reduce the question to the $n$-dimensional Stein-Weiss lemma (see section 2 of Appendix in [13]). This lemma is a natural extension of Theorem 319 in Hardy, Littlewood and Pólya, Inequalities. The advantage of the convolution framework is that sharp constants, non-existence of extremals for infinite measures and simplicity of iteration are attained directly in one step. The role of the Besov norm

$$
C(n, p, \beta) \int_{M \times M} \frac{|v(x)-v(y)|^{p}}{|x-y|^{n+p \beta}} d x d y, \quad v=(-\Delta)^{\alpha} f
$$

with $\operatorname{dim} M=n, 1 \leq p<\infty, \alpha \geq 0,0 \leq \beta<1$, to characterize Sobolev embedding of fractional order in $L^{p}(M)$ grew out of independent work by Aronszajn, Besov, Calderón and Stein (1959-62; see especially [15] and [35]), but also the significant paper by Gagliardo [22]). Here the constant $C(n, p, \beta)$ is chosen to facilitate obtaining $\int|\nabla v|^{p} d x$ in the limit $\beta \rightarrow 1$. It is interesting that (40) is a characteristic example of a larger class of functionals that measure smoothness and are determined by the property of monotonicity under equimeasurable radial decreasing rearrangement (see Theorem 3 in [3]):

$$
\int_{M \times M} \varphi\left[\frac{|f(x)-g(y)|}{\rho(d(x, y))}\right] K[d(x, y)] d x d y
$$

where $M$ is a manifold with distance function $d(x, y)$, and $\varphi, \rho, K$ are non-negative functions on $[0, \infty)$ with the properties: (i) $\varphi(0)=0, \varphi$ convex and monotone increasing; and $t \varphi^{\prime}(t)$ convex; (ii) $\rho$ monotone increasing, $K$ monotone decreasing. Several sharp examples on $S^{n}$ comparing the Besov norm with entropy are calculated in [7]. An interesting implication from this argument is an independent proof of the $L^{2}$ limit of the Hardy-Littlewood-Sobolev inequality on the sphere $S^{n}$. Asymptotic behavior for Besov norm embedding constants is calculated by both Bourgain, Brezis and Mironescu [16, 17] and Maz'ya and Shaposhnikova [29]. In extending that work, interesting new $L^{p}$ Hardy-Rellich inequalities with optimal constants and Besov norms controlling fractional differentiation have recently been obtained by Frank and Seiringer [20, 21]. Stein's ICM lecture at Nice emphasized the importance that analysis on Lie groups would play in future development, including the characteristic example of $S L(2, R)$ and the fundamental role of dilations, but there was not recognition that in going from a manifold to its boundary in the noncompact setting, Hadamard manifolds (e.g., spaces with non-positive

sectional curvature) would have a central place and explicit calculations would need estimates for non-unimodular groups.

\section{APPENDIX}

To make the present discussion more complete, quick calculations are provided to obtain the representation formulas for the fractional Laplacian.

Classical Formula (Aronszajn-Smith). For $f \in \mathcal{S}\left(\mathbb{R}^{n}\right), 0<\alpha<2$

$$
\begin{gathered}
\int_{\mathbb{R}^{n} \times \mathbb{R}^{n}} \frac{|f(x)-f(y)|^{2}}{|x-y|^{n+\alpha}} d x d y=D_{\alpha} \int_{\mathbb{R}^{n}}|\xi|^{\alpha}|\hat{f}(\xi)|^{2} d \xi \\
D_{\alpha}=\frac{4}{\alpha} \pi^{\frac{n}{2}+\alpha} \frac{\Gamma\left(1-\frac{\alpha}{2}\right)}{\Gamma\left(\frac{n+\alpha}{2}\right)}
\end{gathered}
$$


Proof. This is a simple application of the Plancherel theorem (see Stein, page 140).

$$
\begin{aligned}
\int_{\mathbb{R}^{n} \times \mathbb{R}^{n}} \frac{|f(x)-f(y)|^{2}}{|x-y|^{n+\alpha}} d x d y & =\int_{\mathbb{R}^{n}} \frac{1}{|w|^{n+\alpha}}\left[\int_{\mathbb{R}^{n}}|f(x+w)-f(x)|^{2} d x\right] d w \\
& =\int_{\mathbb{R}^{n}} \frac{1}{|w|^{n+\alpha}} \int_{\mathbb{R}^{n}}\left|e^{2 \pi i w \cdot \xi}-1\right|^{2}|\hat{f}(\xi)|^{2} d x d w \\
& =\int_{\mathbb{R}^{n}} \frac{1}{|w|^{n+\alpha}}\left|e^{2 \pi i w \cdot \eta}-1\right|^{2} d w \int_{\mathbb{R}^{n}}|\xi|^{\alpha}|\hat{f}(\xi)|^{2} d \xi
\end{aligned}
$$

with $\eta \in S^{n-1}$. Then

$$
\begin{aligned}
\int_{\mathbb{R}^{n}} \frac{1}{|w|^{n+\alpha}}\left|e^{2 \pi i w \cdot \eta}-1\right|^{2} d w & =2 \int_{\mathbb{R}^{n}} \frac{1}{|w|^{n+\alpha}}(1-\cos 2 \pi w \cdot \eta) d w \\
& =\frac{2 \pi^{\frac{n+\alpha}{2}}}{\Gamma\left(\frac{n+\alpha}{2}\right)} \int_{\mathbb{R}^{n}}(1-\cos 2 \pi w \cdot \eta) \int_{0}^{\infty} t^{\frac{n+\alpha}{2}}-1 e^{-\pi+w^{2}} d t \\
& =\frac{2 \pi^{\frac{n+\alpha}{2}}}{\Gamma\left(\frac{n+\alpha}{2}\right)} \int_{0}^{\infty} t^{\frac{n+\alpha}{2}}-1 \int_{\mathbb{R}^{n}}(1-\cos 2 \pi w \cdot \eta) e^{-\pi t w^{2}} d w \\
& =\frac{2 \pi^{\frac{n+\alpha}{2}}}{\Gamma\left(\frac{n+\alpha}{2}\right)} \int_{0}^{\infty} t^{\frac{\alpha}{2}-1}\left(1-e^{-\pi / t}\right) d t=\frac{2 \pi^{\frac{n+\alpha}{2}}}{\Gamma\left(\frac{n+\alpha}{2}\right)} \int_{0}^{\infty} t^{-\frac{\alpha}{2}-1}\left(1-e^{-t}\right) d t \\
& =\frac{4}{\alpha} \frac{\pi^{\frac{n}{2}+\alpha}}{\Gamma\left(\frac{n+\alpha}{2}\right)} \int_{0}^{\infty} t^{-\alpha / 2} e^{-t} d t=\frac{4}{\alpha} \pi^{\frac{n}{2}+\alpha} \frac{\Gamma\left(1-\frac{\alpha}{2}\right)}{\Gamma\left(\frac{n+\alpha}{2}\right)}
\end{aligned}
$$

The positivity of the integrands justify the exchange of orders of integration using Fubini's theorem. An alternative argument can be given using distribution theory and Green's theorem.

$$
\begin{aligned}
2 \int_{\mathbb{R}^{n}} & \frac{1}{|w|^{n+\alpha}}(1-\cos 2 \pi w \cdot \eta) d w \\
= & {\left[\alpha\left(\frac{n+\alpha}{2}-1\right)\right]^{-1} \int_{\mathbb{R}^{n}} \Delta\left(\frac{1}{|w|^{n+\alpha-2}}\right)(1-\cos 2 \pi w \cdot \eta) d w } \\
& =\left[\alpha\left(\frac{n+\alpha}{2}-1\right)\right]^{-1} \int_{\mathbb{R}^{n}} \frac{1}{|w|^{n+\alpha-2}} \Delta(1-\cos 2 \pi w \cdot \eta) d w \\
& =4 \pi^{2}\left[\alpha\left(\frac{n+\alpha}{2}-1\right)\right]^{-1} \int_{\mathbb{R}^{n}} \frac{1}{|w|^{n+\alpha-2}} \cos 2 \pi w \cdot \eta d w \\
& =4 \pi^{2}\left[\alpha\left(\frac{n+\alpha}{2}-1\right)\right]^{-1} \mathcal{F}\left[\frac{1}{|w|^{n+\alpha-2}}\right](\eta) \\
& =\frac{4 \pi^{\frac{n}{2}+\alpha}}{\alpha} \frac{\Gamma\left(1-\frac{\alpha}{2}\right)}{\Gamma\left(\frac{n+\alpha}{2}\right)}
\end{aligned}
$$


Weighted Formula (Frank-Lieb-Seiringer). For $f \in \mathcal{S}\left(\mathbb{R}^{n}\right), 0<\alpha<\min (2, n)$ and $g(x)=$ $|x|^{\lambda} f(x), 0<\lambda<n-\alpha$

$$
\begin{gathered}
D_{\alpha} \int_{\mathbb{R}^{n}}|\xi|^{\alpha}|\hat{f}(\xi)|^{2} d \xi=\int_{\mathbb{R}^{n} \times \mathbb{R}^{n}} \frac{|g(x)-g(y)|^{2}}{|x-y|^{n+\alpha}}|x|^{-\lambda}|y|^{-\lambda} d x d y \\
+\Lambda(\alpha, \lambda, n) \int_{\mathbb{R}^{n}}|x|^{-\alpha}|f(x)|^{2} d x \\
\Lambda(\alpha, \lambda, n)=\pi^{-\alpha} D_{\alpha}\left[\frac{\Gamma\left(\frac{n-\lambda}{2}\right) \Gamma\left(\frac{\lambda+\alpha}{2}\right)}{\Gamma\left(\frac{\lambda}{2}\right) \Gamma\left(\frac{n-\alpha-\lambda}{2}\right)}\right] \\
D_{\alpha}=\frac{4}{\alpha} \pi^{\frac{n}{2}+\alpha} \frac{\Gamma\left(1-\frac{\alpha}{2}\right)}{\Gamma\left(\frac{n+\alpha}{2}\right)}
\end{gathered}
$$

For $\lambda=\frac{n-\alpha}{2}$, then $\Lambda(\alpha, \lambda, n)=D_{\alpha} / C_{\alpha}$

$$
C_{\alpha}=\pi^{\alpha}\left[\frac{\Gamma\left(\frac{n-\alpha}{4}\right)}{\Gamma\left(\frac{n+\alpha}{4}\right)}\right]^{2}
$$

Proof. Using the classical formula

$$
\begin{aligned}
& D_{\alpha} \int_{\mathbb{R}^{n}}|\xi|^{\alpha}|\hat{f}(\xi)|^{2} d \xi=\int_{\mathbb{R}^{n} \times \mathbb{R}^{n}} \frac{|f(x)-f(y)|^{2}}{|x-y|^{n+\alpha}} d x d y \\
& =\int_{\mathbb{R}^{n} \times \mathbb{R}^{n}} \frac{|g(x)-g(y)|^{2}}{|x-y|^{n+\alpha}}|x|^{-\lambda}|y|^{-\lambda} d x d y+2 \int_{\mathbb{R}^{n} \times \mathbb{R}^{n}}\left[1-\frac{|x|^{\lambda}}{|y|^{\lambda}}\right] \frac{|f(x)|^{2}}{|x-y|^{n+\alpha}} d x d y \\
& =\int_{\mathbb{R}^{n} \times \mathbb{R}^{n}} \frac{|g(x)-g(y)|^{2}}{|x-y|^{n+\alpha}}|x|^{-\lambda}|y|^{-\lambda} d x d y+\Lambda(\alpha, \lambda, n) \int_{\mathbb{R}^{n}}|x|^{-\alpha}|f(x)|^{2} d x \\
& \Lambda(\alpha, \lambda, n)=2 \int_{\mathbb{R}^{n}}\left(1-\frac{1}{|y|^{\lambda}}\right) \frac{1}{|y-\eta|^{n+\alpha}} d y, \quad \eta \in S^{n-1}
\end{aligned}
$$

Note that $\int_{S^{n-1}}\left(1-|y+n|^{-\lambda}\right) d \eta \simeq O\left(|y|^{2}\right)$ as $|y| \rightarrow 0$ so $\Lambda(\alpha, \lambda, n)$ is well-defined for $0<\alpha<2$.

Lemma 15. For $0<\alpha<\min (2, n), 0<\lambda<n-\alpha$ and $\eta \in S^{n-1}$

$$
2 \int_{\mathbb{R}^{n}}\left(1-\frac{1}{|y|^{\lambda}}\right) \frac{1}{|y-\eta|^{n+\alpha}} d y=\pi^{-\alpha} D_{\alpha}\left[\frac{\Gamma\left(\frac{n-\lambda}{2}\right) \Gamma\left(\frac{\lambda+\alpha}{2}\right)}{\Gamma\left(\frac{\lambda}{2}\right) \Gamma\left(\frac{n-\alpha-\lambda}{2}\right)}\right] .
$$

First Proof. For $n \geq 3$, this constant can be calculated using the method of distribution theory, Green's theorem and analytic continuation. Initially let $0<\lambda<n-2$ 


$$
\begin{aligned}
2 \int_{\mathbb{R}^{n}} & \left(1-\frac{1}{|y|^{\lambda}}\right) \frac{1}{|y-\eta|^{n+\alpha}} d y=\frac{2}{\alpha(n+\alpha-2)} \int_{\mathbb{R}^{n}}\left(1-\frac{1}{|y|^{\lambda}}\right) \Delta\left(|y-\eta|^{-n-\alpha+2}\right) d y \\
& =\left[\alpha\left(\frac{n+\alpha}{2}-1\right)\right]^{-1} \int \Delta\left(1-\frac{1}{|y|^{\lambda}}\right)|y-\eta|^{-n-\alpha+2} d y \\
& =\lambda(n-2-\lambda)\left[\alpha\left(\frac{n+\alpha}{2}-1\right)\right]^{-1} \int_{\mathbb{R}^{n}}|y|^{-\lambda-2}|y-\eta|^{-n-\alpha+2} d y \\
& =\lambda(n-2-\lambda)\left[\alpha\left(\frac{n+\alpha}{2}-1\right)\right]^{-1}\left(|y|^{-\lambda-2} *|y|^{-n-\alpha+2}\right)(\eta) \\
& =\pi^{n / 2} \lambda(n-2-\lambda)\left[\alpha\left(\frac{n+\alpha}{2}-1\right)\right]^{-1}\left[\frac{\Gamma\left(\frac{n-2-\lambda}{2}\right) \Gamma\left(\frac{2-\alpha}{2}\right) \Gamma\left(\frac{\lambda+\alpha}{2}\right)}{\left.\Gamma\left(\frac{\lambda+2}{2}\right) \Gamma\left(\frac{n+\alpha-2}{2}\right) \Gamma\left(\frac{n-\alpha-\lambda}{2}\right)\right]}\right. \\
= & \frac{4 \pi^{n / 2}}{\alpha}\left[\frac{\Gamma\left(\frac{n-\lambda}{2}\right) \Gamma\left(1-\frac{\alpha}{2}\right) \Gamma\left(\frac{\lambda+\alpha}{2}\right)}{\Gamma\left(\frac{\lambda}{2}\right) \Gamma\left(\frac{n+\alpha}{2}\right) \Gamma\left(\frac{n-\alpha-\lambda}{2}\right)}\right] \\
= & \pi^{-\alpha} D_{\alpha}\left[\frac{\Gamma\left(\frac{n-\lambda}{2}\right) \Gamma\left(\frac{\lambda+\alpha}{2}\right)}{\Gamma\left(\frac{\lambda}{2}\right) \Gamma\left(\frac{n-\alpha-\lambda}{2}\right)}\right] \cdot
\end{aligned}
$$

Since both the beginning and final terms are analytic in $\lambda$ on the strip $0<R e \lambda<n-\alpha$, this formula extends by analytic continuation to hold on that region for $n \geq 3$ :

$$
2 \int_{\mathbb{R}^{n}}\left(1-\frac{1}{|y|^{\lambda}}\right) \frac{1}{|y-\eta|^{n+\alpha}} d y=\pi^{-\alpha} D_{\alpha}\left[\frac{\Gamma\left(\frac{n-\lambda}{2}\right) \Gamma\left(\frac{\lambda+\alpha}{2}\right)}{\Gamma\left(\frac{\lambda}{2}\right) \Gamma\left(\frac{n-\alpha-\lambda}{2}\right)}\right] .
$$

Set $\lambda=\sigma+\frac{n-\alpha}{2}$; then for $-\left(\frac{n-\alpha}{2}\right)<\sigma<\frac{n-\alpha}{2}$

$$
\Lambda\left(\alpha, \sigma+\frac{n-\alpha}{2}, \eta\right)=\pi^{-\alpha} D_{\alpha}\left[\frac{\Gamma\left(\frac{n+\alpha}{4}-\frac{\sigma}{2}\right) \Gamma\left(\frac{n+\alpha}{4}+\frac{\sigma}{2}\right)}{\Gamma\left(\frac{n-\alpha}{4}+\frac{\sigma}{2}\right) \Gamma\left(\frac{n-\alpha}{4}-\frac{\sigma}{2}\right)}\right] .
$$

Observe that $\Lambda\left(\alpha, \sigma+\frac{n-\alpha}{2}, n\right)$ as a function of $\sigma$ is symmetric and has a negative second derivative so $\Lambda$ is concave in $\sigma$ for the allowed range and has a maximum at $\sigma=0$ or $\lambda=$ $(n-\alpha) / 2$. Hence

$$
\Lambda(\alpha, \lambda, n) \leq \pi^{-\alpha} D_{\alpha}\left[\frac{\Gamma\left(\frac{n+\alpha}{4}\right)}{\Gamma\left(\frac{n-\alpha}{4}\right)}\right]^{2}=D_{\alpha} / C_{\alpha} .
$$

Second Proof. To give a full proof of the lemma, an integral representation is used for the factor $1-|y|^{-\lambda}$ :

$$
1-|y|^{-\lambda}=\frac{\pi^{\lambda / 2}}{\Gamma(\lambda / 2)} \int_{0}^{\infty} t^{\frac{\lambda}{2}-1}\left(e^{-\pi t}-e^{-\pi t|y|^{2}}\right) d t .
$$


Then

$$
\begin{aligned}
& 2 \int_{\mathbb{R}^{n}}\left(1-|y|^{-\lambda}\right)|y-\eta|^{-n-\alpha} d y \\
& =\frac{2 \pi^{\lambda / 2}}{\Gamma(\lambda / 2)} \int_{\mathbb{R}^{n}} \int_{0}^{\infty} t^{\frac{\lambda}{2}-1}\left(e^{-\pi t}-e^{-\pi t|y|^{2}}\right) d t|y-\eta|^{-n-\alpha} d y \\
& =\frac{2 \pi^{\lambda / 2}}{\Gamma(\lambda / 2)} \int_{0}^{\infty} t^{\frac{\lambda}{2}-1} \int_{\mathbb{R}^{n}}\left(e^{-\pi t}-e^{-\pi t|y|^{2}}\right)|y-\eta|^{-n-\alpha} d y d t \\
& =\frac{2 \pi^{\lambda / 2}}{\alpha(n+\alpha-2) \Gamma\left(\frac{\lambda}{2}\right)} \int_{0}^{\infty} t^{\frac{\lambda}{2}-1} \int_{\mathbb{R}^{n}}\left(e^{-\pi t}-e^{-\pi t|y|^{2}}\right) \Delta\left(|y-\eta|^{-n-\alpha+2}\right) d y d t \\
& =\frac{2^{\pi^{\lambda / 2}}}{\alpha(n+\alpha-2) \Gamma\left(\frac{\lambda}{2}\right)} \int_{0}^{\infty} t^{\frac{\lambda}{2}-1} \int_{\mathbb{R}^{n}} \Delta\left(-e^{-\pi t|y|^{2}}\right)|y-\eta|^{-n-\alpha+2} d y d t \\
& =\frac{4 \pi^{\frac{n}{2}+\frac{\lambda}{2}+\alpha} \Gamma\left(\frac{1-\alpha}{2}\right)}{\alpha \Gamma\left(\frac{\lambda}{2}\right) \Gamma\left(\frac{n+\alpha}{2}\right)} \int_{0}^{\infty} t^{\frac{\lambda}{2}-\frac{n}{2}-1} \int_{\mathbb{R}^{n}} e^{2 \pi i \xi \cdot \eta} e^{-\pi|\xi|^{2} / t}|\xi|^{\alpha} d \xi d t \\
& =D_{\alpha} \frac{\pi^{\lambda / 2}}{\Gamma\left(\frac{\lambda}{2}\right)} \int_{\mathbb{R}^{n}} e^{2 \pi i \xi \cdot \eta} \int_{0}^{\infty} t^{\frac{\lambda}{2}-\frac{n}{2}-1} e^{-\pi|\xi|^{2} / t} d t|\xi|^{\alpha} d \xi \\
& =D_{\alpha} \frac{\pi^{\lambda / 2}}{\Gamma\left(\frac{\lambda}{2}\right)} \int_{\mathbb{R}^{n}} e^{2 \pi i \xi \cdot \eta} \int_{0}^{\infty} t^{\frac{n-\lambda}{2}-1} e^{-\pi t|\xi|^{2}} d t|\xi|^{\alpha} d \xi \\
& =D_{\alpha} \pi^{-\frac{n}{2}+\lambda} \frac{\Gamma\left(\frac{n-\lambda}{2}\right)}{\Gamma\left(\frac{\lambda}{2}\right)} \int_{\mathbb{R}^{n}} e^{2 \pi i \xi \cdot \eta} \frac{1}{|\xi|^{n-\alpha-\lambda}} d \xi \\
& =D_{\alpha} \pi^{-\alpha} \frac{\Gamma\left(\frac{\alpha+\lambda}{2}\right)}{\Gamma\left(\frac{n-\alpha-\lambda}{2}\right)} \frac{\Gamma\left(\frac{n-\lambda}{2}\right)}{\Gamma\left(\frac{\lambda}{2}\right)}=\Lambda(\alpha, \lambda, n) \text {. }
\end{aligned}
$$

The last exchange of orders of integration requires that the calculation be done in the context of distributions. Different proofs are given in [19].

In terms of the modulus of continuity, the Aronszajn-Smith formula can be extended to larger values of $\alpha$ (see Stein, pages 140, 162-163).

Classical Formula (Stein). For $f \in \mathcal{S}\left(\mathbb{R}^{n}\right), 0<\alpha<4$

$$
\begin{gathered}
\int_{\mathbb{R}^{n} \times \mathbb{R}^{n}} \frac{|f(x+y)+f(x-y)-2 f(x)|^{2}}{|y|^{n+\alpha}} d x d y=E_{\alpha} \int_{\mathbb{R}^{n}}|\xi|^{\alpha}|\hat{f}(\xi)|^{2} d \xi \\
E_{\alpha}=\frac{4-2^{\alpha}}{2-\alpha} \frac{8}{\alpha} \pi^{\frac{n}{2}+\alpha} \frac{\Gamma\left(2-\frac{\alpha}{2}\right)}{\Gamma\left(\frac{n+\alpha}{2}\right)} .
\end{gathered}
$$


Proof. Apply the Plancheral formula

$$
\begin{aligned}
\int_{\mathbb{R}^{n} \times \mathbb{R}^{n}} & \frac{|f(x+y)+f(x-y)-2 f(x)|^{2}}{|y|^{n+\alpha}} d x d y \\
= & \int_{\mathbb{R}^{n}} \frac{1}{|y|^{n+\alpha}} \int_{\mathbb{R}^{n}}\left|e^{2 \pi i y \cdot \xi}+e^{-2 \pi i y \cdot \xi}-2\right|^{2}|\hat{f}(\xi)|^{2} d \xi d y \\
= & \int_{\mathbb{R}^{n}}|\hat{f}(\xi)|^{2} \int_{\mathbb{R}^{n}}\left|e^{2 \pi i y \cdot \xi}+e^{-2 \pi i y \cdot \xi}-2\right|^{2} \frac{1}{|y|^{n+\alpha}} d y d \xi \\
= & \int_{\mathbb{R}^{n}} \frac{1}{|y|^{n+\alpha}}\left|e^{2 \pi i y \cdot \eta}+e^{-2 \pi i y \cdot \eta}-2\right|^{2} d y \int_{\mathbb{R}^{n}}|\xi|^{\alpha}|\hat{f}(\xi)|^{2} d \xi
\end{aligned}
$$

for $\eta \in S^{n-1}$. Observe that

$$
\begin{aligned}
& \left|e^{2 \pi i y \cdot \eta}+e^{-2 \pi i y \cdot \eta}-2\right|^{2}=4|1-\cos 2 \pi y \cdot \eta|^{2} \\
& =8(1-\cos 2 \pi y \cdot \eta)-2(1-\cos 4 \pi y \cdot \eta) .
\end{aligned}
$$

Using the previous calculation of $D_{\alpha}, E_{\alpha}=\left(4-2^{\alpha}\right) D_{\alpha}$ and

$$
E_{\alpha}=\frac{4-2^{\alpha}}{2-\alpha} \frac{8}{\alpha} \pi^{\frac{n}{2}+\alpha} \frac{\Gamma\left(2-\frac{\alpha}{2}\right)}{\Gamma\left(\frac{n+\alpha}{2}\right)} .
$$

This result leads to three interesting formulas:

$$
\begin{gathered}
\int_{\mathbb{R}^{n} \times \mathbb{R}^{n}} \frac{|f(x+y)+f(x-y)-2 f(x)|^{2}}{|y|^{n+2}} d x d y=\frac{\ln 2}{n} \frac{2 \pi^{n / 2}}{\Gamma(n / 2)} \int_{\mathbb{R}^{n}}|\nabla f|^{2} d x \\
\int_{\mathbb{R}^{n} \times \mathbb{R}^{n}} \frac{|f(x+y)+f(x-y)-2 f(x)|^{2}}{|y|^{n+\alpha}} d x d y \geq \frac{E_{\alpha}}{C_{\alpha}} \int_{\mathbb{R}^{n}}|x|^{-\alpha}|f(x)|^{2} d x
\end{gathered}
$$

for $0<\alpha<\min (4, n)$ and

$$
C_{\alpha}=\pi^{\alpha}\left[\Gamma\left(\frac{n-\alpha}{4}\right) / \Gamma\left(\frac{n+\alpha}{4}\right)\right]^{2}
$$

and

(iii) $\int_{\mathbb{R}^{n} \times \mathbb{R}^{n}} \frac{|f(x+y)+f(x-y)-2 f(x)|^{2}}{|y|^{n+\alpha}} d x d y \geq \int_{\mathbb{R}^{n} \times \mathbb{R}^{n}} \frac{\left|f^{*}(x+y)+f^{*}(x-y)-2 f^{*}(x)\right|^{2}}{|y|^{n+\alpha}} d x d y$

for $0<\alpha \leq 2$ and $f^{*}$ is the equimeasurable radial decreasing rearrangement of $|f|$ on $\mathbb{R}^{n}$.

\section{ACKNOWLEDGEMENT}

I would like to thank Michael Perelmuter for drawing my attention to the arguments of Herbst [24] and Kovalenko-Perelmuter-Sememov [26], Sharif Nasibov for reference [31, and Emanuel Carneiro for helpful comments. I am very indebted to Eli Stein for the many ideas he shared in his marvelous lectures on Fourier Analysis in the fall of 1969 in new Fine Hall and in his mimeographed notes which were to become his classic text on Singular Integrals. Some of these ideas return in this paper. 


\section{REFERENCES}

[1] N. Aronszajn and K.T. Smith, Theory of Bessel potentials I, Ann. Inst. Fourier 11 (1961), 385-475.

[2] W. Beckner, Inequalities in Fourier Analysis, Annuals Math. 102 (1975), 159-182.

[3] W. Beckner, Sobolev inequalities, the Poisson semigroup and analysis on the sphere $S^{n}$, Proc. Nat. Acad. Sci. 89 (1992), 4816-4819.

[4] W. Beckner, Sharp Sobolev inequalities on the sphere and the Moser-Trudinger inequality, Annals Math. 138 (1993), 213-242.

[5] W. Beckner, Geometric inequalities in Fourier analysis, Essays on Fourier Analysis in Honor of Elias M. Stein, Princeton University Press, 1995, pp. 36-68.

[6] W. Beckner, Pitt's inequality and the uncertainty principle, Proc. Amer. Math. Soc. 123 (1995), $1897-1905$.

[7] W. Beckner, Logarithmic Sobolev inequalities and the existence of singular integrals, Forum Math. 9 (1997), 303-323.

[8] W. Beckner, Sharp inequalities and geometric manifolds, J. Fourier Anal. Appl. 3 (1997), 825-836.

[9] W. Beckner Geometric asymptotics and the logarithmic Sobolev inequality, Forum Math. 11 (1999), 305-337.

[10] W. Beckner, On the Grushin operator and hyperbolic symmetry, Proc. Amer. Math. Soc. 129 (2001), 12331246.

[11] W. Beckner, Estimates on Moser embedding, Potential Analysis 20 (2004), 345-359.

[12] W. Beckner, Weighted inequalities and Stein-Weiss potentials, Forum Math. 20 (2008), 587-606.

[13] W. Beckner, Pitt's inequality with sharp convolution estimates, Proc. Amer. Math. Soc. 136 (2008), 18711885.

[14] W. Beckner, On Maz'ya-Sobolev embedding and hyperbolic symmetry (in preparation).

[15] O. V. Besov, Investigation of a family of function spaces in connection with theorems of imbedding and extension, Trudy Mat. Inst. Steklov 60 (1961), 42-81; Amer. Math. Soc. Transl. 40 (1964), 85-126.

[16] J. Bourgain, H. Brezis and P. Mironescu, Another look at Sobolev spaces, Optimal control and partial differential equations - innovations and applications, IOS Press, Amsterdam, 2000, pp. 439-455.

[17] J. Bourgain, H. Brezis and P. Mironescu, Limiting embedding theorems for $W^{s, p}$ when $s \uparrow 1$ and applications, J. Anal. Math. 87 (2002), 77-101.

[18] S. Eilertsen, On weighted fractional integral inequalities, J. Funct. Anal. 185 (2001), 342-366.

[19] R. L. Frank, E. H. Lieb and R. Seiringer, Hardy-Lieb-Thirring inequalities for fractional Schrödinger operators, J. Amer. Math. Soc. 21 (2008), 925-950.

[20] R. L. Frank and R. Seiringer, Non-linear ground state representations and sharp Hardy inequalities, J. Funct. Anal. 25 (2008), 3407-3430.

[21] R. L. Frank and R. Seiringer, Sharp fractional Hardy inequalities in half-spaces (arXiv: 0906.1561).

[22] E. Gagliardo, Caratterizzazoni delletracce sulla frontiera relative ad alcune classi di funzioni in $n$ variabiliti, Rend. Sem. Math. Padova 27 (1957), 284-305.

[23] G. H. Hardy, J. E. Littlewood and G. Pólya, Inequalities, Cambridge University Press, 1952.

[24] I. W. Herbst, Spectral theory of the operator $\left(p^{2}+m^{2}\right)^{1 / 2}-Z e^{2} / r$, Comm. Math. Phys. 53 (1977), 285-294.

[25] N. Karapetiants and S. Samko, Equations with involutive operators, Birkhäuser, 2001.

[26] V. F. Kovalenko, M. A. Perelmuter and Ya. A. Sememov, Schrödinger operators with $L_{w}^{1 / 2}\left(\mathbb{R}^{\ell}\right)-$ potentials, J. Math. Phys. 22 (1981), 1033-1044.

[27] E.H. Lieb, Sharp constants in the Hardy-Littlewood-Sobolev and related inequalities, Annals Math. 118 (1983), 349-374.

[28] V. G. Maz'ya, Sobolev spaces, Springer-Verlag, 1985.

[29] V. G. Maz'ya and T. Shaposhnikova, On the Bourgain, Brezis and Mironescu theorem concerning limiting embeddings of fractional Sobolev spaces, J. Funct. Anal. 195 (2002), 230-238.

[30] B. Sz. Nagy, Über integratungleichungen zwischen einer funktion und ihrer ableitung, Acta Sci. Math. (Szeged) 10 (1941), 64-74.

[31] Sh. M. Nasibov, Optimal constants in some Sobolev inequalities and their applications to the nonlinear Schrödinger equation, Dokl. Akad. Nauk SSSR 307 (1989), 538-542.

[32] H. R. Pitt, Theorems on Fourier series and power series, Duke Math. J. 3 (1937), 747-755.

[33] S. Samko, Best constant in the weighted Hardy inequality: the spatial and spherical version, Fract. Calc. Anal. Appl. 8 (2005), 39-52.

[34] E. M. Stein, Interpolation of linear operators, Trans. Amer. Math. Soc. 83 (1956), 482-492.

[35] E. M. Stein, The characterization of functions arising as potentials, Bull. Amer. Math. Soc. 68 (1962), $577-582$.

[36] E. M. Stein, Singular integrals and differentiability properties of functions, Princeton University Press, 1970. 
[37] E. M. Stein, Some problems in harmonic analysis suggested by symmetric spaces and semi-simple groups, Actes Congrès Intern. Math. (Nice, 1970), Tome 1, 173-189.

[38] E. M. Stein and G. Weiss, Interpolation of operators with change of measures, Trans. Amer. Math. Soc. 87 (1958), 159-172.

[39] E. M. Stein and G. Weiss, Fractional integrals on n-dimensional Euclidean space, J. Math. Mech. 7 (1958), 503-514.

[40] A. Weil, L'integration dans les groupes topologiques et ses applications, Hermann, 1940.

[41] M. I. Weinstein, Nonlinear Schrödinger equations and sharp interpolation estimates, Comm. Math. Phys. 87 (1983), 567-576.

[42] D. Yafaev, Sharp constants in the Hardy-Rellich inequalities, J. Funct. Anal. 168 (1999), $121-144$.

[43] A. Zygmund, Trigonometric series, Cambridge University Press, 1959.

Department of Mathematics, The University of Texas at Austin, 1 University Station C1200, AUsTin TX 78712-0257 USA

E-mail address: beckner@math.utexas.edu 